\title{
An improved method for growing neurons: comparison with standard methods
}

Pozzi Diletta $^{{ }^{*}}$, Ban Jelena ${ }^{1,2^{*}}$, Iseppon Federico ${ }^{1}$ and Torre Vincent ${ }^{1}$.

${ }^{1}$ Neurobiology Sector, International School for Advanced Studies (SISSA), via Bonomea 265, 34136 Trieste, Italy.

${ }^{2}$ Department of biotechnology, University of Rijeka, Radmile Matejčić 2, 51000 Rijeka, Croatia.

*These authors contribute equally to this work.

Correspondence and requests for materials should be addressed to V.T. (email: torre@sissa.it). 

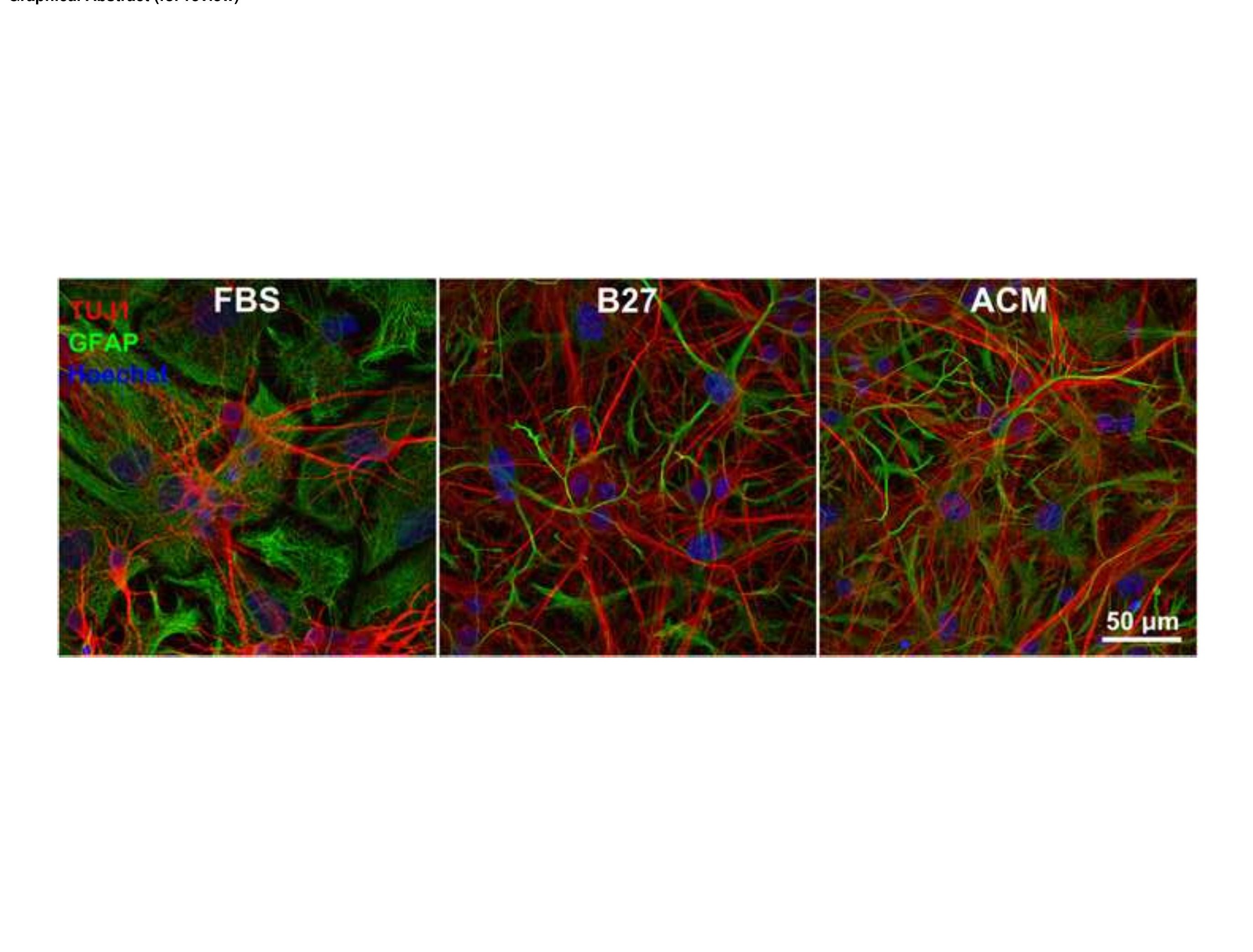

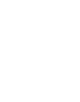

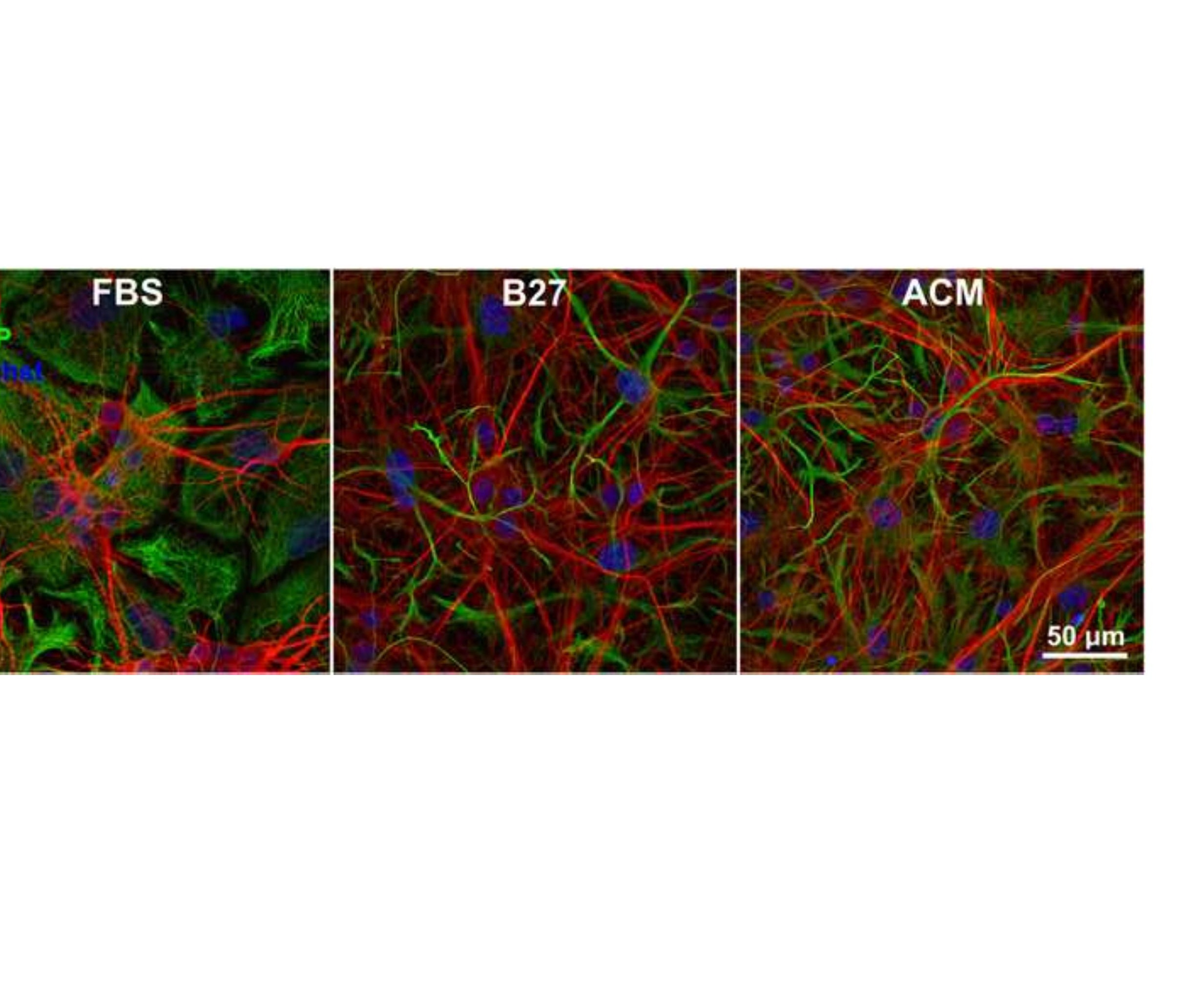

 


\section{Highlights}

- An improved protocol for primary hippocampal cell cultures is proposed.

- The method relies on serum-free astrocytes conditioned medium (ACM).

- The ACM method is extensively compared with other two commonly used protocols.

- ACM improved morphology and function of both short- and long-term cultures. 


\title{
An improved method for growing neurons: comparison with standard protocols
}

\author{
Diletta Pozzi ${ }^{1}$, Jelena Ban ${ }^{1,2^{*}}$, Federico Iseppon ${ }^{1}$ and Vincent \\ Torre ${ }^{1}$.
}

${ }^{1}$ Neurobiology Sector, International School for Advanced Studies (SISSA), via Bonomea 265, 34136 Trieste, Italy. ${ }^{2}$ Department of Biotechnology, University of Rijeka, Radmile Matejčić 2, 51000 Rijeka, Croatia.

*These authors contributed equally to this work. Correspondence and requests for materials should be addressed to V.T. (email: torre@sissa.it).

\section{Abstract}

Background: Since different culturing parameters - such as media composition or cell density - lead to different experimental results, it is important to define the protocol used for neuronal cultures. The vital role of astrocytes in maintaining homeostasis of neurons - both in vivo and in vitro - is well established: the majority of improved culturing conditions for primary dissociated neuronal cultures rely on astrocytes.

New Method: Our culturing protocol is based on a novel serum-free preparation of astrocyte conditioned medium (ACM). We compared the proposed ACM culturing method with other two commonly used methods: Neurobasal/B27- and FBS- based media. We performed morphometric characterization by immunocytochemistry and functional analysis by calcium imaging for all three culture methods at 1,7,14 and 60 days in vitro (DIV).

Results: ACM-based cultures gave the best results for all tested criteria, i.e. growth cone's size and shape, neuronal outgrowth and branching, network activity and synchronization, maturation and long-term survival. The differences were more pronounced when compared with FBS-based medium. Neurobasal/B27 cultures were comparable to ACM for young cultures (DIV1), but not for culturing times longer than DIV7.

Comparison with Existing Method(s): ACM-based cultures showed more robust neuronal outgrowth at DIV1. At DIV7 and 60, the activity of neuronal network grown in ACM had a more vigorous spontaneous electrical activity and a higher degree of synchronization.

Conclusions: We propose our ACM-based culture protocol as an improved and more suitable method for both short- and long-term neuronal cultures.

\section{Keywords}

Neuronal culture - Astrocyte - B27 Supplement - Fetal Bovine Serum - Neuronal branching Calcium signaling.

\section{Introduction}


Primary dissociated neuronal cultures, obtained from embryonic or postnatal rodent brain regions such as the hippocampus or the cortex, represent a well-established in vitro model for studying neuronal networks, both on a short (network formation) and a long term (differentiation, maturation) scales ${ }^{1,2}$. When cells are dissociated, plated on appropriate substrates and cultured in appropriate media, they grow processes and form ex-novo a functional network.

Several media (N2, DMEM/F12) supplemented with serum, most commonly from fetal bovine (FBS) or horse origin, are commonly used for neuronal cultures. However, the chemical composition of the animal serum is not fully defined and includes some factors not present in the brain; moreover, the production of commercial serum is prone to batch to batch variability ${ }^{3,4}$. For these reasons, the use of a chemically defined, serum-free medium is recommended for studies in which a complete control of the environment in the nutrient medium is desired ${ }^{5}$. Several attempts were made in order to find serum free - media for optimal neuronal growth. Currently, the most widely used is Neurobasal medium with B27 supplement ${ }^{6,7}$. Moreover, several modifications to Neurobasal/B27 were proposed with the addition/modification of glycoproteins, lipid and hormone molecules (NbActiv4, NS21) ${ }^{8-10}$. Most of those protocols were designed to promote long-term neuronal survival in an almost pure neuronal culture: neurons of embryonic (E18) and postnatal (P0) origin were cultured in the presence of $<1 \%$ and $6-8 \%$ of glial cells, respectively ${ }^{7,11}$.

However, the role of astrocytes for maintaining the homeostasis of neuronal cells both in vivo and in vitro has been clear for a long time ${ }^{12-16}$. Different options are now available for a successful hippocampal cell culture, all of them involving astrocytes: I- direct plating of dissociated neurons on a glial feeder layer ${ }^{17}$, II- suspension of the coverslip with plated neurons above a glial feeder layer $^{18}$ and III- culturing neurons in astrocyte-conditioned medium (ACM). This third method has an advantage: it does not require a feeder cell's layer while providing all the soluble factors released by astrocytes, such as growth factors, signaling molecules and lipids ${ }^{19-21}$.

ACM has been demonstrated to increase neuronal survival and stem cell differentiation ${ }^{22-24}$. However, the use of ACM in cell culturing protocols is still uncommon and often limited to in vitro models of pathologies, where its protective effect on neurons following mechanical injury and hypoxia has been demonstrated ${ }^{18}$. Standard neuronal culturing methods are mostly based on serumenriched or serum-free, supplement-enriched media ${ }^{7}$, both of which rely on commercially available resources. ACM, instead, can be obtained by the researcher from the same animal species used for primary neuronal cell's cultures.

Our goal is to establish an optimal, easy to use and reproducible culturing protocol for obtaining viable, functionally connected and long - term surviving primary hippocampal cultures. For this purpose, we compared two traditional culturing protocols with a new method based on the use of ACM as a growing medium. Our method differs from other previously published protocols ${ }^{24}$ because the medium is produced in house and it is totally serum - free, unlike the commercial ACM obtained by conditioning of traditional astrocyte medium (supplemented with 10\% FBS).

Our comparison is based on a morphometric and functional characterization of neuronal networks grown in the three different media. Above all, we describe a serum -free culturing method for long - term neuronal cultures with preserved functionality and connectivity, reminiscent of that seen in the intact tissue. The three culturing protocols that we compared were: I- FBS based medium (traditionally used in our $1 \mathrm{lab}^{26}$ and by other research groups ${ }^{17,27,28}$; II- Neurobasal/B27 medium as described in Beaudoin ${ }^{7}$; III- our serum - free ACM medium. The exact composition for all the three growing media is reported in the Methods section and the conditions are referred to as FBS, B27 and ACM, respectively. The comparison between the three protocols was carried out by maintaining the same experimental parameters (cell density, animal age and functional tests). 
Therefore, the differences in morphology and electrical activity are attributed to the effect of the different media.

\section{Results}

\subsection{Setting of the plating protocol}

We analyzed the morphology, electrical activity and long-term survival of our cell cultures grown in the three different media. All other parameters such as coverslip coating, plating medium and cells density were the same in the three cases. Briefly, after dissociation, the cells were suspended in plating medium (Neuronal medium supplemented with 10\% FBS, see Methods and Supplementary Table 1 for the complete formulation), seeded at a density of 100,000 cells/sample and incubated at $37^{\circ} \mathrm{C}$ for half an hour in order to allow the cell's attachment on the coverslips previously coated with polyornithine (see Methods). After that, the sample was divided in three groups - in this work referred to as FBS, B27 and ACM - and added to the appropriate growing medium. We used the same plating medium in the three cases because when cells were plated in serum-free Neurobasal/B27 medium, cells death increased and no electrical activity was detectable. Interestingly, when plated in ACM medium, the cells were able to survive for more than one month with consistent electrical activity (Supplementary Video 1).

\subsection{Growth cones' morphology}

Twenty-four hours after plating, we analyzed the cell morphology by immunocytochemistry. Cells were fixed and stained for filamentous actin (F-actin) and for $\beta$-tubulin III, a marker for postmitotic neurons. At a first inspection, we observed a higher cell growth in Neurobasal/B27-based media, compared to FBS at DIV1. In particular, neuronal growth cones (GCs) were larger, thicker, richer in F-actin and had a more elaborated morphology with many filopodia (Figure 1A-C). To quantify those differences, we measured and compared the surface of GCs among the three conditions. Confocal images were acquired with slice spacing of $0.2 \mu \mathrm{m}$, up to $5 \mu \mathrm{m}$ z-stack thickness, and for each GC a maximum intensity projection of the stacks for F-actin was used. The contours of each GCs were manually traced and the surface was measured using ImageJ/Fiji. At least $20 \mathrm{GCs}$ for each condition were analyzed (see Methods). The GCs in Neurobasal/B27-based media were significantly larger comparing to FBS. GCs in ACM, in particular, showed the biggest size; however, the difference between ACM and B27 was not statistically significant $\left(36.27 \pm 1,69 \mu \mathrm{m}^{2}\right.$ in FBS, 86.27 $\pm 10.63 \mu \mathrm{m}^{2}$ in B27 and 102.19 $\pm 8.60 \mu \mathrm{m}^{2}$ in ACM; Figure 1D).

A similar analysis was done by counting the number of filopodia in each GC (Fig. 1F): GCs in FBS-based medium had on average $9.48 \pm 0.62$ filopodia, B27 $19.72 \pm 1$ while ACM $24.24 \pm 1.76$, matching the most complex morphology observed in B27-based media (examples of elaborated forms - never observed in FBS - are shown in Supplementary Figure 1). 

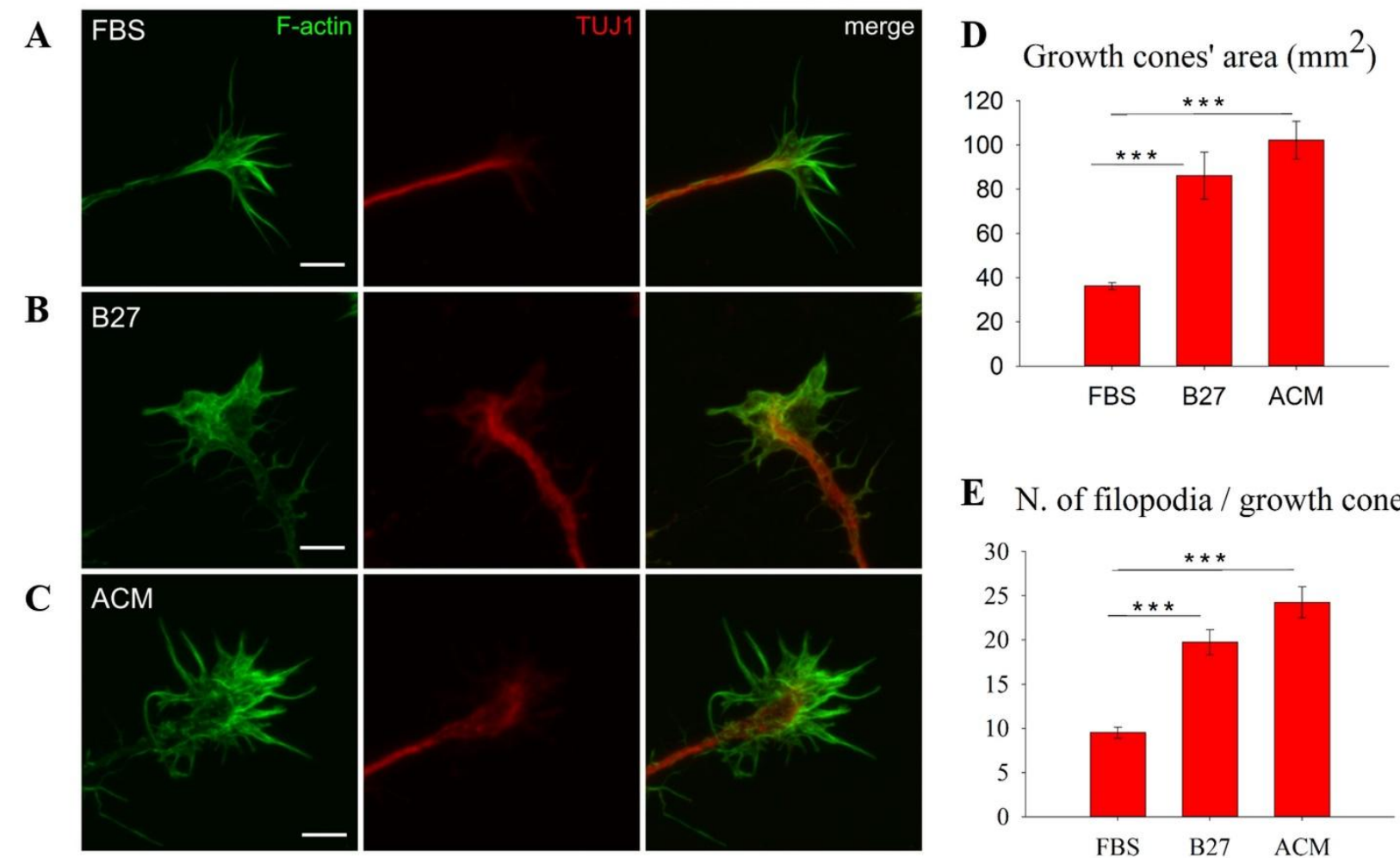

E N. of filopodia / growth cone

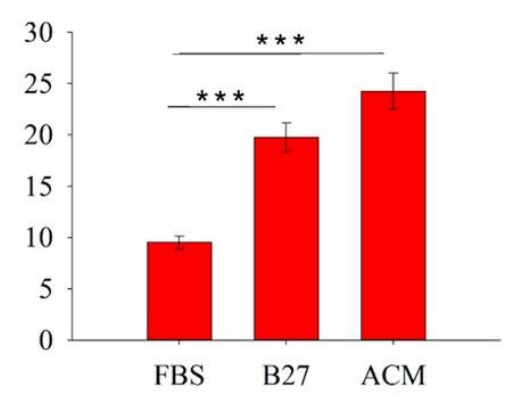

Figure 1. Neuronal growth cones at DIV1for FBS (A), B27 (B) and ACM (C). Cells were stained for F-actin (left), $\beta$ tubulin III (TUJ1, middle) and merged (right). (D) Growth cone surfaces for the three conditions tested. (E) Number of filopodia per growth cone for FBS, B27 and ACM respectively. Scale bar, $5 \mu \mathrm{m} . \mathrm{n}=25$ growth cones for FBS, $\mathrm{n}=29$ growth cones for B27, $\mathrm{n}=25$ growth cones for ACM. *** $\mathrm{p}<0.001$ One - Way ANOVA, Tukey post - hoc test.

\subsection{Neurite outgrowth}

Next, we analyzed the neurite outgrowth/branching. At DIV1, the overall morphology of neurons in Neurobasal/B27-based media was more elaborated and neurites in particular were thicker and had more spines (Figure 2A-C). On the other hand, neurons in FBS had thinner neurites, simpler morphology, tended to grow straight and to cluster. To quantify the differences in the three conditions we considered, the same procedure was used: at DIV1, cells were fixed, stained and confocal images were acquired using a tubulin marker to calculate the average number of neurites per each neuron. FBS cultures had the lowest number $(2.87 \pm 1.45, \mathrm{n}=205$ neurons analyzed $)$ comparing to the Neurobasal/B27-based media $(5.30 \pm 2.61$ for B27 and 5.44 \pm 3.06 for ACM, respectively; $n=214$ neurons in B27 and $n=202$ neurons in ACM, Table 1). As for GCs, the ACM condition resulted in higher branching (up to 19 neurites per neuron, compared to maximal numbers of 9 and 13 for FBS and B27, respectively; Table 1) although not significantly different when compared to B27. These results confirmed that Neurobasal/B27-based media promote extensive neurite outgrowth resulting in higher branching of neurons. 

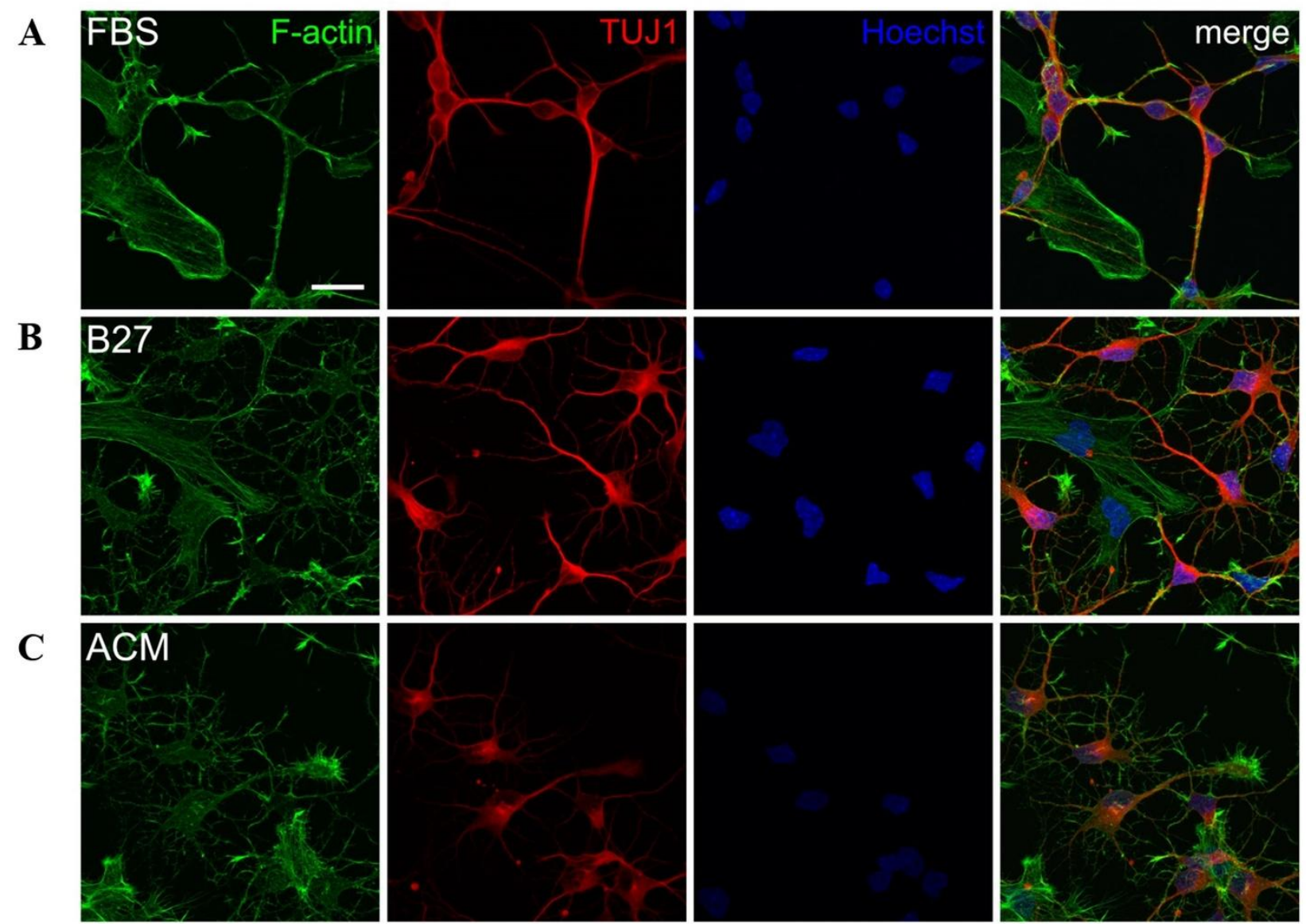

Figure 2. Neuronal branching at DIV1for FBS (A), B27 (B) and ACM (C). From left to right: cells were stained for Factin (green), $\beta$-tubulin III (TUJ1, red), Hoechst nuclear marker (blue) and then merged (right). Z-stacks of about $10 \mu \mathrm{m}$ with $0.2 \mu \mathrm{m}$ step size were acquired and maximum intensity projections of confocal images are here shown. Scale bar, $20 \mu \mathrm{m}$.

\begin{tabular}{|l|l|l|l|}
\hline & Neurites/cell & Cells analyzed & $\begin{array}{l}\text { Max number of } \\
\text { neurites/cells }\end{array}$ \\
\hline FBS & $2,87 \pm 0,10$ & 205 & 9 \\
\hline B27 & $5,30 \pm 0,18 * * *$ & 214 & 13 \\
\hline ACM & $5,44 \pm 0,22 * * *$ & 202 & 19 \\
\hline
\end{tabular}

Table 1. Number of neurites per cell at DIV1 in FBS, B27 and ACM represented as mean \pm standard error of the mean. Significantly higher values were observed in B27 and ACM comparing to FBS. *** p $<0,001$. One - Way ANOVA, Tukey post - hoc test.

\subsection{Astrocytes morphology and percentage}

We did not observe significant differences in glial cells' morphology at this early developmental stage (DIV1, Supplementary Figure 2). At DIV7, astrocytes grown in FBS and stained for glial fibrillary acidic protein (GFAP) assumed a flattened morphology (Fig. 3A). In Neurobasal/B27 media instead, complex shapes were observed with several cellular processes closely associated with neurons (Fig. 3B and C). Moreover, we compared the proportion of astrocytes between the three protocols. At DIV7, the percentage of glia in the neuronal cultures was estimated by counting GFAP - positive cells and observed to be around 32\% in FBS, 23\% in B27 and 29\% in ACM (Table 2). As expected, because of the presence of several growth factors ${ }^{19,21}$, FBS and ACM 
resulted in higher percentages, despite the addition of the proliferation blocker cytosine- $\beta$-Darabinofuranoside (Ara-C) at DIV2 (see Methods). The morphological differences were progressively more pronounced with the extension of the culturing time required for glial maturation. Astrocytes in ACM and B27 develop long and branched processes typical of a more mature morphology compared to the astrocytes in FBS at DIV14 (Supplementary Figure 3).
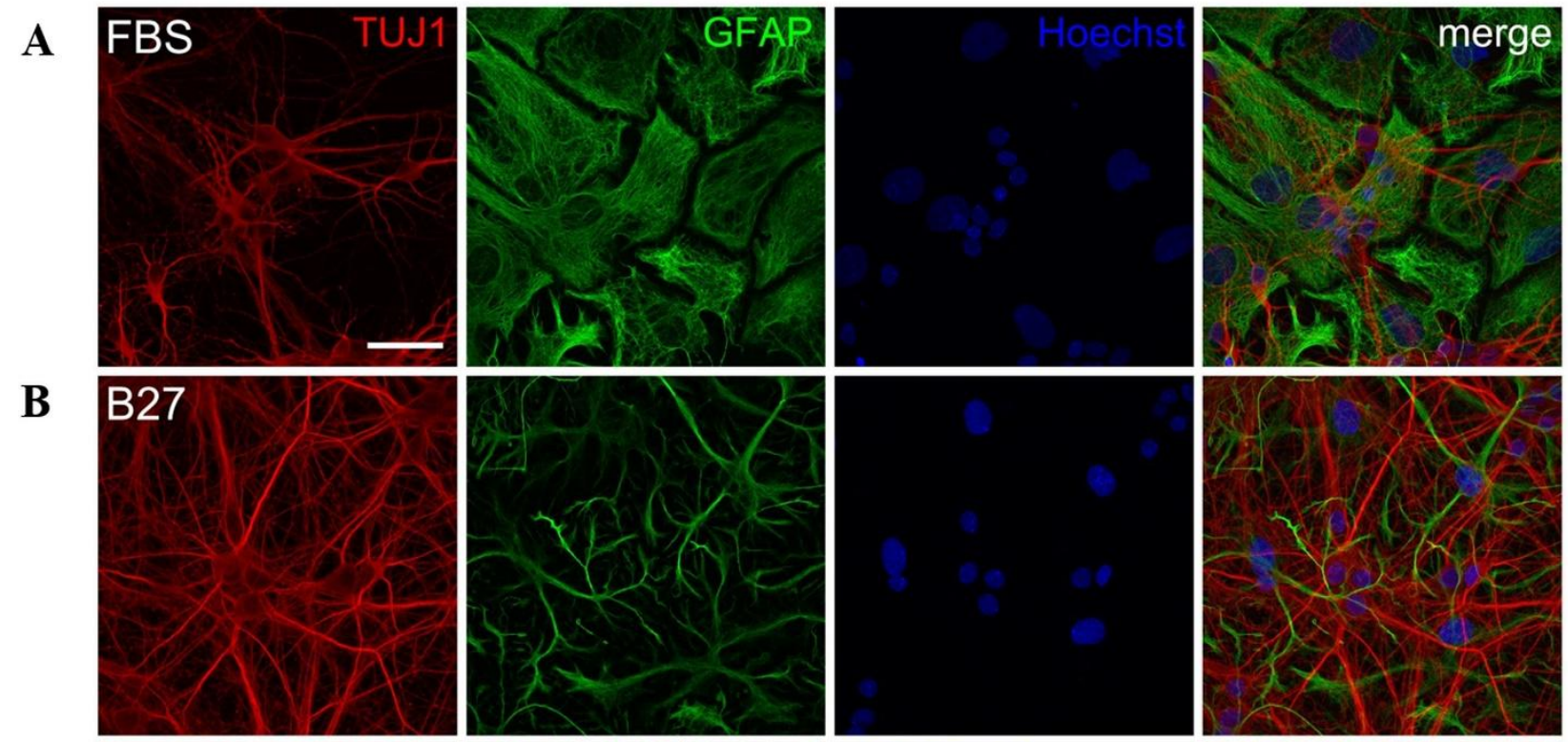

\section{ACM}
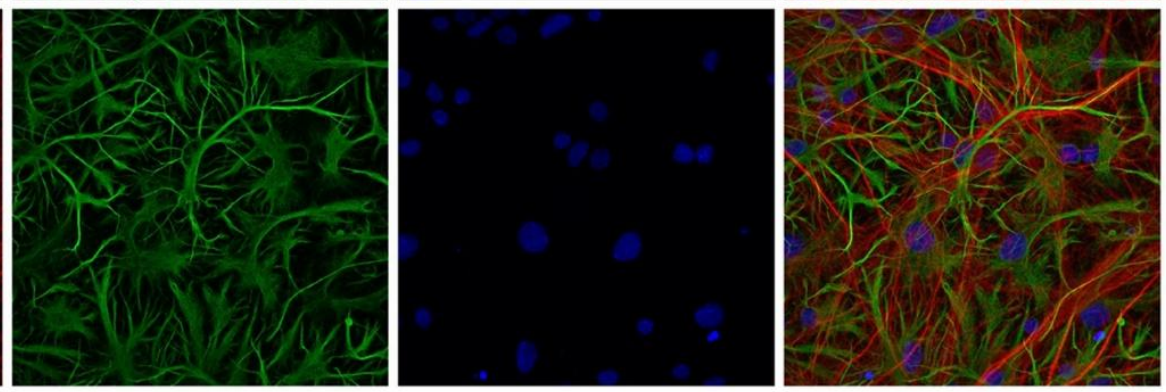

Figure 3. Neurons and glial cells morphology at DIV7 for FBS (A), B27 (B) and ACM (C). From left to right: cells were stained for $\beta$-tubulin III (TUJ1, red), GFAP (green), Hoechst nuclear staining (blue), and then merged. Confocal maximum intensity projections images of about $15 \mu \mathrm{m}$-thick $\mathrm{z}$ stacks acquired with $0,5 \mu \mathrm{m}$ step size are here shown. Scale bar, $50 \mu \mathrm{m}$.

\begin{tabular}{|l|l|l|}
\hline & \% glia & N. cells analyzed \\
\hline FBS & $31,92 \pm 2,48$ & 704 \\
\hline B27 & $23,02 \pm 2,02$ & 648 \\
\hline ACM & $29,16 \pm 3,11$ & 506 \\
\hline
\end{tabular}

Table 2. Percentage of glia in the neuronal culture \pm standard error of the mean in the three conditions at DIV7, with the corresponding number of cells analyzed. 


\subsection{Functional properties of neuronal networks}

Next we investigated the functional differences among neuronal networks. The spontaneous electrical activity of the neurons was analyzed by measuring their calcium transients $\left(\mathrm{DF} / \mathrm{F}_{0}\right)^{29}$, obtained by acquiring fluorescence images at $3-10 \mathrm{~Hz}$ for $10 \mathrm{~min}$ in every field of view. The onset time of calcium transients was defined by detecting those events in the fluorescence signal that exceeded at least by three times the standard deviation of the noise (about $0.01 \mathrm{DF} / \mathrm{F}_{0}$, see Methods). Moreover, we added tetrodotoxin (TTX) to the dish at the end of the recording (Supplementary Figure 4) and verified the disappearance of calcium signals in the majority of cells in the culture, that were identified as neurons. The remaining cells were identified as astrocytes and excluded from the analysis, since they were showing calcium signals after TTX addition in the typical form of calcium waves ${ }^{30}$.

Fluorescent images of the three cultures at DIV7, loaded with Oregon Green 488 BAPTA -1 $®$ cellpermeable calcium dye, and their representative traces are shown in Fig. 4A-C. The frequency of calcium peaks (Fig. 4D) showed an increasing trend from FBS $(0.020 \pm 0.002$ peaks/s) to B27 $(0.026 \pm 0.002$ peaks $/ \mathrm{s})$ and ACM $(0.0322 \pm 0.0004$ peaks/s $)$ with significant differences between FBS and ACM. Moreover, the mean time interval between calcium peaks (Inter Event Interval, IEI, Fig. 4E) was measured for every neuronal trace in order to analyze not only the frequency but also the distribution of peaks over time: the lowest value was observed in the ACM condition, with significant differences compared to FBS; an increase in the IEI was observed in B27 compared to $\mathrm{ACM}$, although not significant $(63.99 \pm 4.73 \mathrm{~s}$ for FBS; $57.06 \pm 9.53 \mathrm{~s}$ for B27; $37.88 \pm 2.80 \mathrm{~s}$ for ACM). Neurons grown in ACM showed a more homogeneous distribution of IEI comparing to the other conditions, as shown in Fig. 4G. Finally, the average correlation index (Fig. 4F) between the calcium peaks of different cells was calculated from cross correlation raster plots (see Methods) and showed a significantly higher value in ACM $(0.684 \pm 0.004)$ when compared to both FBS $(0.430 \pm 0.005)$ and $\mathrm{B} 27(0.614 \pm 0.012)$; a significantly higher value was also found in B27 compared to FBS ( $n=157(5)$ couples of neurons (cultures) for FBS; $n=647(4)$ couples of neurons (cultures) for B27; $\mathrm{n}=1680$ (4) couples of neurons (cultures) for ACM). Taken together, these data show that ACM cultures have higher frequency and synchronous activity compared to both FBS and $\mathrm{B} 27$. 

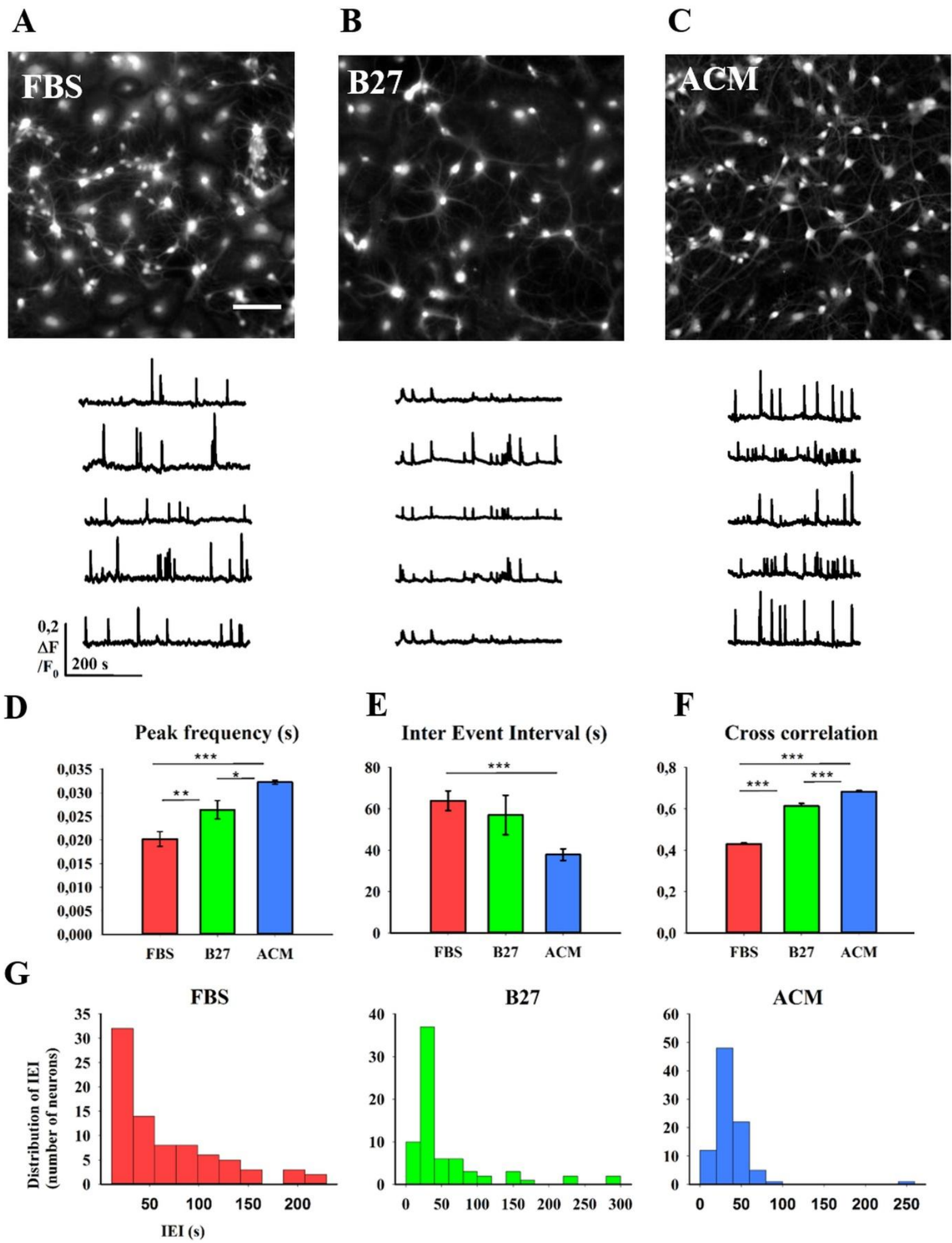

Figure 4. Fluorescent images of neuronal cultures at DIV7 in FBS (A), B27 (B) and ACM (C) loaded with $4 \mu \mathrm{M}$ Oregon Green 488 BAPTA - 1® and corresponding calcium traces of 5 representative neurons simultaneously recorded. Average frequency (D), Inter Event Interval (E) and cross correlation index (F) of neuronal calcium peaks in the three conditions. Distribution of Inter Event Interval (IEI) in the three conditions (G). Scale bar, $100 \mu \mathrm{m}$. $\mathrm{n}=121 \mathrm{neurons}$ for FBS; $\mathrm{n}=71$ neurons for B27; $\mathrm{n}=98$ neurons for ACM. $* \mathrm{p}<0.05 * * \mathrm{p}<0.01 * * * \mathrm{p}<0.001$ One-way ANOVA, Tukey post-hoc test). 
Subsequently, we performed the same experiment with neuronal cultures at mature stages, i.e. 2 months growth (Fig. 5). The overall pattern of spontaneous electrical activity was found to be similar to the previously examined time point. The average frequency of calcium peaks (Fig. 5D) was still significantly increased in ACM when compared to the other two conditions, while a difference between B27 and FBS was no longer observed $(0.063 \pm 0.006$ peaks/s in FBS; $0.058 \pm$ 0.005 peaks/s in B27; $0.091 \pm 0.005$ peaks/s in ACM). An increased IEI (Figure 5E) was found in FBS compared to both B27 - based media $(24.45 \pm 2.49 \mathrm{~s}$ in FBS; $23.03 \pm 2.67 \mathrm{~s}$ in B27; $17.35 \pm$ $1.45 \mathrm{~s}$ in ACM). The distribution of IEI at this point was instead similar in the three conditions (Fig. 5G). Finally, a significantly higher cross correlation index (Fig. 5F) was measured in both B27 based media compared to FBS, and in ACM compared to B27 (0.62 for 363(4) couples of neurons (cultures) in FBS; 0.77 for 504(4) couples of neurons (cultures) in B27; 0.83 for 844(4) couples of neurons (cultures) in ACM). Thus, the neuronal cultures grown in ACM maintain an overall higher spontaneous electrical activity and higher degree of synchrony over time compared to the cultures grown in traditional media. These results are consistent with previous works that demonstrate the advantage of using ACM for growing long - term cell cultures ${ }^{24}$. 

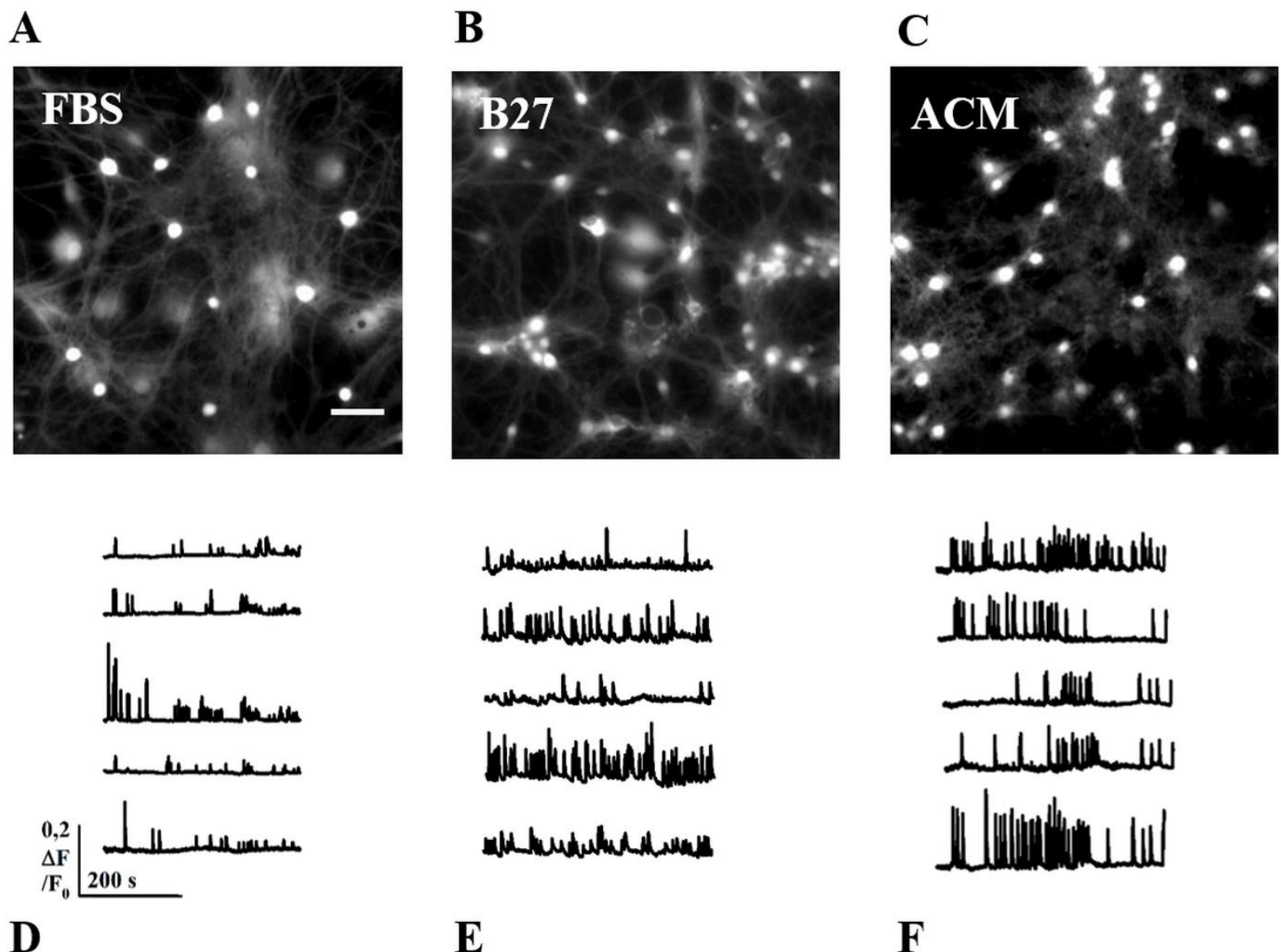

E

F

Peak frequency $(s)$
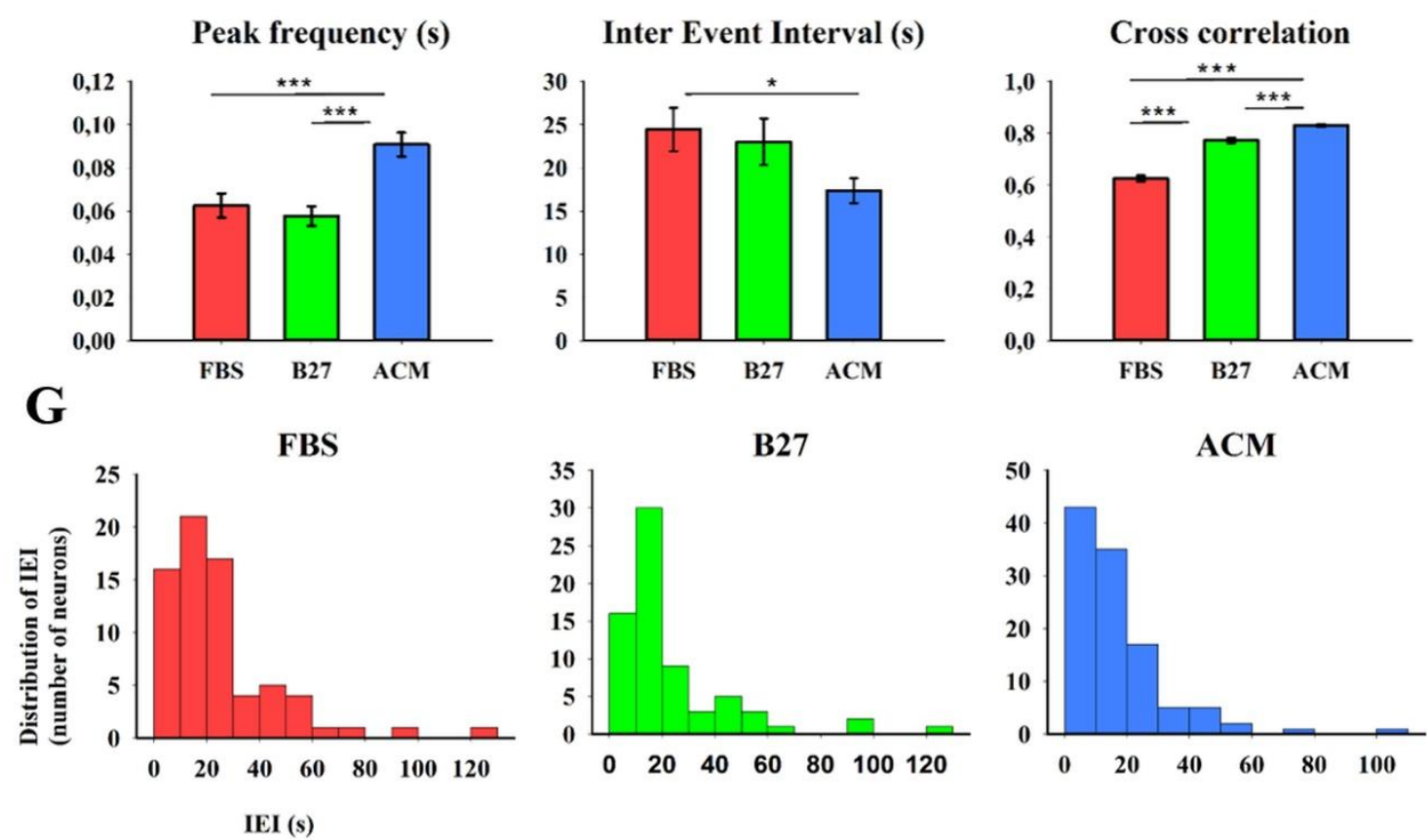

Figure 5. Fluorescent images of neuronal cultures grown for 2 months in FBS (A), B27 (B) and ACM (C) loaded with $4 \mu \mathrm{M}$ Oregon Green 488 BAPTA - 1, AM ${ }^{\circledR}$ and corresponding calcium traces of 5 representative neurons simultaneously recorded. Average frequency (D), Inter Event Interval (E) and cross correlation index (F) of neuronal calcium peaks in the three conditions. Distribution of Inter Event Interval (IEI) in the three conditions (G). Scale bar, $100 \mu \mathrm{m} . \mathrm{n}=71$ neurons for FBS; $\mathrm{n}=70$ neurons for B27; $\mathrm{n}=110$ neurons for ACM. $* \mathrm{p}<0.05 * * \mathrm{p}<0.001$ One-way ANOVA, Tukey post-hoc test. 


\subsection{Cell survival and morphology in the long - term cultures}
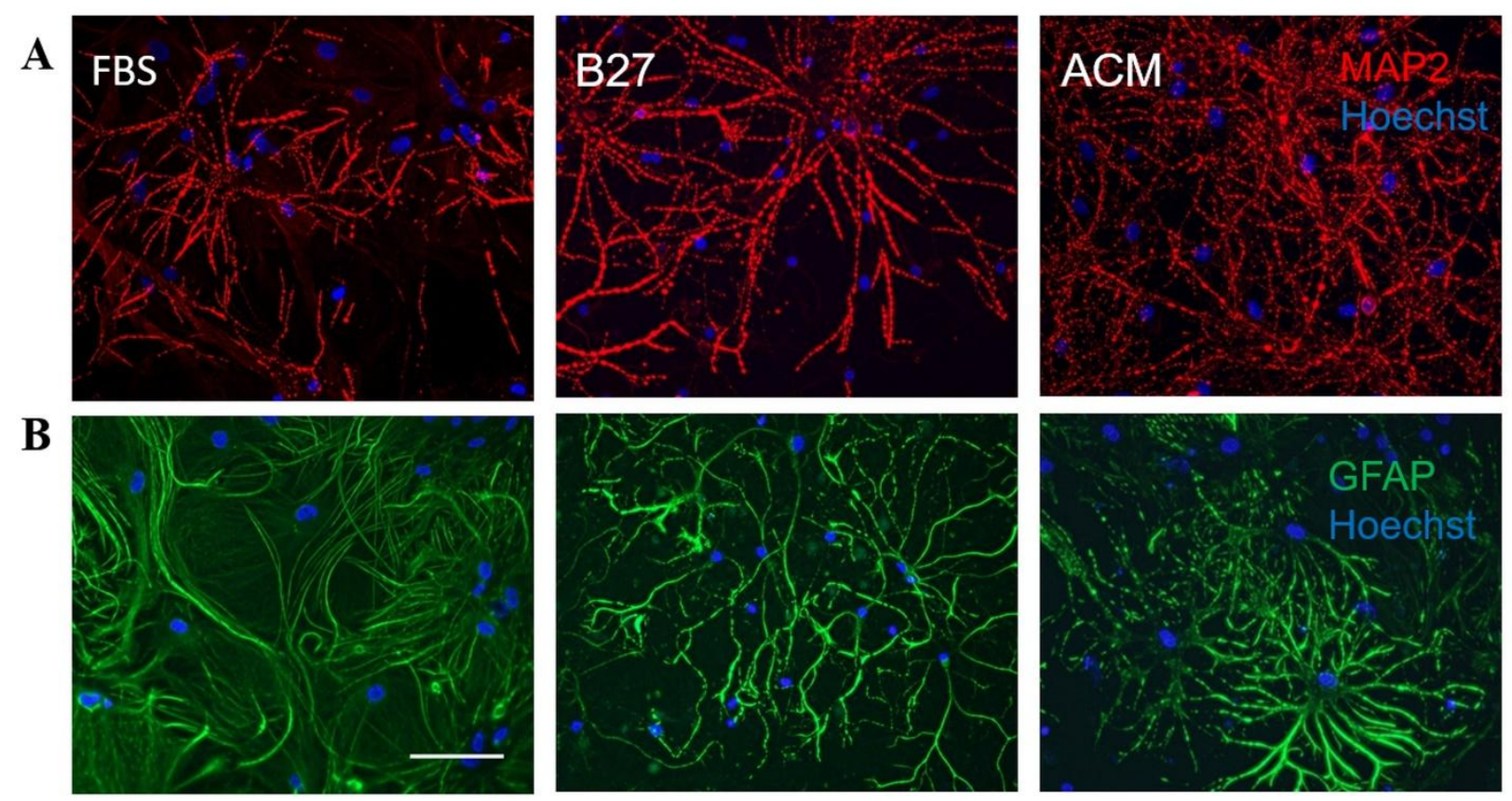

Figure 6. Morphology of neurons (A) and astrocytes (B) grown for 2 months in FBS (left), B27 (middle) and ACM (right). Cells were stained for MAP2 (red), GFAP (green) and Hoechst nuclear staining (blue).

Finally, we investigated the cell morphology of the three different cultures by immunocytochemistry. Neurons were stained with microtubule associated protein (MAP) 2 and astrocytes with GFAP (Fig. 6A-B). After 2 months, ACM cultures showed a denser neuronal network with higher cell survival, while in both FBS and B27 we observed a progressive increase of cell-free surface, most probably due to the loss of neurons. Astrocytes in FBS maintained a relatively simple, undifferentiated morphology with low degree of arborization. In contrast, GFAP staining in B27 revealed thin and long astrocytes processes. ACM cultures maintained a highly branched and complex phenotype, similar to mature astrocytes observed in vivo ${ }^{31}$.

\section{Discussion}

Our aim is to establish a reproducible and easy - to - use protocol for growing long - term neuronal cultures with healthy and almost physiological functional properties. For this purpose, we produced a serum -free, Neurobasal/B27 - based ACM for growing primary hippocampal neurons directly after plating, unlike other methods in which neurons are first grown in serum - enriched media ${ }^{32}$. Unlike commercially available astrocyte - conditioned media, our ACM is derived from the same animal species and strain used for primary neuronal preparations and contains no additives. We harvested ACM as a growing medium, which allows a controlled percentage of glia in the culture. In this way, we preserved the physiological crosstalk between neurons and glia cells without using the glial feeder layer. 
The resulting hippocampal cultures in ACM showed more robust neuronal outgrowth and branching compared to those grown in a serum - enriched medium, which makes them suitable for developmental studies as they provide an in vitro model reminiscent of the physiological CNS. Even though little is known about the in vivo morphology of axonal and dendritic growth cones, some works report evidence that the structure of neuronal growth cones is elaborated with numerous filopodia, especially at early developmental stages ${ }^{33}$. Astrocytes grown in serum -free conditions show a complex morphology that better represents in vivo systems ${ }^{31,34}$ comparing to the flat astrocytic cell's bodies observed in FBS (compare astrocytes morphology between ACM and FBS in Fig.3 and Supplementary Fig.3). Indeed, the fibroblast-like morphology of astrocytes is known as an artifact due to the presence of serum in the culturing medium ${ }^{35,36}$. Astrocytes in the hippocampus of adult rats show instead numerous branches and processes as previously demonstrated ${ }^{37}$.

In addition to the improved short-term cultures, we obtained long-term neuronal cultures with preserved functional connectivity up to two months, as demonstrated by calcium imaging experiments. Different culturing conditions, i. e. physical properties and chemical composition of the medium used, may affect the affinity of the dye for calcium ions and the fluorescence intensity itself $^{38}$. In order to minimize these effects, we chose Oregon Green ${ }^{\circledR} 488$ BAPTA-1, AM calcium dye because of its high-affinity and high signal-to-noise ratio ${ }^{39}$. Moreover, the same experimental conditions such as dye concentration, temperature and incubation time were maintained for all the three protocols tested. A similar level of baseline fluorescence intensity was observed by visual inspection when recording cells grown in the three culturing media. Therefore, we assume that the uptake of the calcium sensitive dye from both neurons and astrocytes was similar in the three protocols, mainly dependent on the hydrophobicity of the AM - conjugated molecule ${ }^{40}$. However, further investigations are required to explain the role of astrocytes secreted factors in modulation of calcium-mediated neuronal processes ${ }^{41}$.

The presence of the differentiating factor B27 leads to an overall increase of the network's electrical activity when compared to what happens in the serum - based medium. However, the addition of ACM to the Neurobasal/B27 medium resulted in a further amplification of the overall spontaneous activity compared to both traditional methods (see Fig. 4-5). In particular, neurons grown in ACM showed significantly higher degree of correlation both in the short - and in the long - term compared to FBS and B27, suggesting that the synchronizing effect attributed to astrocytes may reside in their soluble factors ${ }^{42,43}$. Moreover, we demonstrated that ACM can totally replace the use of serum, even at the plating stage, while maintaining viability and functionality of the network (Supplementary Video 1). Although Neurobasal/B27 is the most commonly used serum-free medium for neuronal cultures (so called "maintenance medium"7) it still requires the presence of FBS in the plating medium. Finally, since ACM alone is supposed to provide all the nutrients for long - term neuronal survival (e.g. lipids, BDNF and thrombospondins ${ }^{19-21}$ ), the use of additional growth factors in the culturing medium is not necessary.

\section{Conclusions}

We conclude that our ACM method is an improved and more suitable culture protocol for both short - and long - term studies, resulting in cell cultures that represent a realistic model of the CNS, useful for investigating the mechanisms of neuronal maturation and degeneration. 


\section{Materials and Methods}

\subsection{Hippocampal cultures}

Hippocampal neurons from Wistar rats (P2-P3) were prepared in accordance with the guidelines of the Italian Animal Welfare Act, and their use was approved by the Local Veterinary Service, the SISSA Ethics Committee board and the National Ministry of Health (Permit Number: 630-III/14) in accordance with the European Union guidelines for animal care (d.1.116/92; 86/609/C.E.). The animals were anaesthetized with $\mathrm{CO}_{2}$ and sacrificed by decapitation, and all efforts were made to minimize suffering. Glass coverslips $(15 \mathrm{~mm}$ diameter) were coated with $50 \mu \mathrm{g} / \mathrm{ml}$ poly-L-ornithine (Sigma-Aldrich, St. Louis, MO, USA) overnight, and just before cells' seeding, a thin layer of Matrigel (diluted 1:50 with culture medium; Corning, Tewksbury MA, USA) was applied. Dissociated cells were resuspended in Neuronal medium with the following composition: minimum essential medium (MEM) with GlutaMAX ${ }^{\mathrm{TM}}$ supplemented with $10 \%$ dialyzed fetal bovine serum (FBS, all from Thermo Fisher Scientific, Waltham, MA, USA), 0.6\% D-glucose, $15 \mathrm{mM}$ Hepes, 0.1 $\mathrm{mg} / \mathrm{ml}$ apo-transferrin, $30 \mu \mathrm{g} / \mathrm{ml}$ insulin, $0.1 \mu \mathrm{g} / \mathrm{ml}$ D-biotin, $1 \mu \mathrm{M}$ vitamin $\mathrm{B} 12$ and $2.5 \mu \mathrm{g} / \mathrm{ml}$ gentamycin (all from Sigma-Aldrich). A drop containing 100,000 cells was deposited per each 15 $\mathrm{mm}$ diameter coverslip and incubated for $30 \mathrm{~min}$ at $37^{\circ} \mathrm{C}$. For each coverslip, placed in a 12-well tissue culture plate, $1.5 \mathrm{ml}$ of culture medium was then added. Three sets of media were used: $\mathbf{I}$ Neuronal medium with 10\% FBS (the same used for resuspending cells, see above), II - Neurobasal medium supplemented with $\mathrm{B} 27$ and GlutaMAX ${ }^{\mathrm{TM}}$ according to the manufacturer's instructions (all from Thermo Fisher) and $2.5 \mu \mathrm{g} / \mathrm{ml}$ gentamycin, III- ACM (see below) in 1:1 ratio with Neurobasal/B27 medium. Half of the medium was changed after $48 \mathrm{~h}$ and $2 \mu \mathrm{M}$ cytosine- $\beta$-Darabinofuranoside (Ara-C; Sigma-Aldrich) was added to all culture media. The concentration of FBS (in medium containing 10\% FBS) was decreased to 5\%. Half of the medium was changed then twice per week. The neuronal cultures were maintained in an incubator at $37^{\circ} \mathrm{C}, 5 \% \mathrm{CO}_{2}$ and $95 \%$ relative humidity. For a detailed description of the chemical composition of Neuronal vs Neurobasal medium, see Supplementary Table 1.

\subsection{Glia and ACM preparation}

Glial cells were prepared as described in Kaech and Baker ${ }^{15}$ with some modifications. Cortices from P2-P3 Wistar rats were dissociated as described for hippocampal cultures. Cells were plated in $15 \mu \mathrm{g} / \mathrm{ml}$ poly-L-ornithine (Sigma-Aldrich) coated tissue-culture flasks at the density of $7.5 \times 10^{6}$ cells per $75 \mathrm{~cm}^{2}$ flask in Dulbecco's minimum essential medium (DMEM) with GlutaMAX ${ }^{\mathrm{TM}}$ (Thermo Fisher Scientific) supplemented with $10 \%$ foetal bovine serum (FBS) and Penicillin/Streptomycin (all from Euroclone, Pero, Italy). The next day the medium was changed in order to remove cell debris and loosely attached cells. Cells were cultured for $\sim 1$ week and medium was changed every 2-3 days. When almost confluent, cells were replated in $10 \mathrm{~cm}$ diameter Petri dishes at the density of $5 \times 10^{5}$ cells per dish and expanded until they reached $75 \%$ confluence. The medium was then replaced with Neurobasal medium supplemented with B27 and GlutaMAX ${ }^{\mathrm{TM}}$ (all from ThermoFisher) and $2.5 \mu \mathrm{g} / \mathrm{ml}$ gentamycin (Sigma-Aldrich). After $48 \mathrm{~h}$ ACM was collected, filtered and stored at $4^{\circ} \mathrm{C}$.

\subsection{Immunocytochemistry and Imaging}

Cells were fixed in $4 \%$ paraformaldehyde containing $0.15 \%$ picric acid in phosphate-buffered saline (PBS), saturated with $0.1 \mathrm{M}$ glycine, permeabilized with $0.1 \%$ Triton $\mathrm{X}-100$, saturated with $0.5 \%$ BSA (all from Sigma-Aldrich) in PBS and then incubated for 1h with primary mouse monoclonal antibodies: glial fibrillary acidic protein (GFAP, Sigma-Aldrich), anti- $\beta$-tubulin III (TUJ1, Covance, Berkeley, CA) and mouse monoclonal anti-microtubule associated protein 2 (MAP2, SigmaAldrich). The secondary antibodies were goat anti-mouse Alexa Fluor ${ }^{\circledR}$ 594, goat anti-mouse 
immunoglobulin (Ig) $G_{1}$ Alexa Fluor ${ }^{\circledR} 488$ and goat anti-mouse $\operatorname{IgG}_{2 a}$ Alexa Fluor ${ }^{\circledR}$ 594. F-actin was marked with Alexa Fluor $488^{\circledR}$ phalloidin (all from Thermo Fisher Scientific) and the incubation time was $30 \mathrm{~min}$. Nuclei were stained with $2 \mu \mathrm{g} / \mathrm{ml}$ in PBS Hoechst 33342 (SigmaAldrich) for $5 \mathrm{~min}$. All the incubations were performed at room temperature $\left(20-22^{\circ} \mathrm{C}\right)$. The cells were examined using a Leica DMIRE2 confocal microscope (Leica Microsystems GmbH, Wetzlar, Germany) equipped with DIC and fluorescence optics, diode laser $405 \mathrm{~nm}$, Ar/ArKr 488nm and $\mathrm{He} / \mathrm{Ne} 543 / 594 \mathrm{~nm}$ lasers. 63x magnification and a $1.4 \mathrm{NA}$ objective was used. Images were acquired at $1024 \times 1024$ pixels resolution and $\mathrm{z}$ stacks with $200-250 \mathrm{~nm}$ steps. In addition, Leica DM6000 fluorescent microscope equipped with DIC and fluorescence optics, CCD camera and Volocity 5.4 3D imaging software (PerkinElmer, Coventry, UK) were used for cell counting experiments. The fluorescence images were collected with a 20x magnification and 0.5 NA objective. For each image at least 30 slices were acquired with slice spacing of $0.5 \mu \mathrm{m}$. Image $\mathrm{J}$ by W. Rasband (developed at the U.S. National Institutes of Health and available at http://rsbweb.nih.gov/ij/) was used for image processing.

\subsection{Calcium imaging}

The cells were incubated with the non-ratiometric calcium dye Oregon Green ${ }^{\circledR} 488$ BAPTA-1, AM, (Thermo Fisher Scientific) dissolved in anhydrous DMSO (Sigma-Aldrich) at a concentration of 4 $\mathrm{mM}$ (stock solution), with the addition of Pluronic F-127 20\% solution in dimethyl sulfoxide (DMSO, Thermo Fisher Scientific) for increasing the cell's permeability. The two components were dissolved at a ratio of 1:1 in Ringer's solution $(145 \mathrm{mM} \mathrm{NaCl}, 3 \mathrm{mM} \mathrm{KCl}, 1.5 \mathrm{mM} \mathrm{CaCl}, 1 \mathrm{mM}$ $\mathrm{MgCl}_{2}, 10 \mathrm{mM}$ glucose and $10 \mathrm{mM}$ Hepes, $\mathrm{pH}$ 7.4) to give a final dye concentration of $4 \mu \mathrm{M}$, and incubated at $37^{\circ} \mathrm{C}$ for $30 \mathrm{~min}$. The cultures were then transferred to a glass-bottom Petri dish in order to allow visualization in a Nikon Eclipse Ti-U inverted microscope equipped with an HBO 103 W/2 mercury short arc lamp (Osram, Munich, Germany), a mirror unit (exciter filter BP 465 $495 \mathrm{~nm}$, dichroic $505 \mathrm{~nm}$, emission filter BP 515-555) and an Electron Multiplier CCD Camera C9100-13 (Hamamatsu Photonics, Japan). The experiments were performed at RT for 20 $\mathrm{min} / \mathrm{sample}$. At the end of each registration, the antagonist of voltage - gated sodium channels tetrodotoxin (TTX, Sigma - Aldrich) was added to the culture at a concentration of $1 \mu \mathrm{M}$ and the sample was recorded for additional 5 min.

Images were acquired using the NIS Element software (Nikon, Japan) with an S-Fluor 20x/0.75 NA objective at a sampling rate of 3-10 Hz. The spatial resolution was $256 \times 256$ pixels. To avoid saturation of the signals, excitation light intensity was attenuated by ND4 and ND8 neutral density filters (Nikon).

\subsection{Data analysis}

\subsubsection{Morphometric analysis, neurite and cell counting}

At least three different preparations were used. For neurite counting, nuclear stain was used to acquire randomly 3-5 fields with comparable cell densities (approx. 30 cells/field). TUJ-stained neurites were counted and a mean number of neurites per neuron was calculated for all three tested conditions.

\subsection{2 $\mathrm{Ca}^{2+}$ imaging processing and analysis}

As described in our previous work $^{26,44}$, the initial video was processed with the ImageJ (U. S. National Institutes of Health, Bethesda, MA) software. Briefly, neurons were localized by selecting the cells bodies with a region of interest (ROI), and an additional ROI was selected to subtract the background. The fluorescence intensity over time, If $(\mathrm{t})$, of every selected ROI was displayed and 
the extent of its decay - consequence of dye bleaching - was evaluated. To compensate photo bleaching, the decay of If(t) was fitted with a cubic or polynomial spline (Y(t)) interpolating If $(t)$ at 10 or 20 points. The function $\mathrm{Y}(t)$ fitting $\operatorname{If}(t)$ was then added to the original optical signal - to compensate dye bleaching - and the fractional optical signal was taken as DF/F $=\mathrm{Y}(t)+\operatorname{If}(t)) / \operatorname{If}(0)$, where If $(0)$ is the fluorescence intensity at the beginning of the recording.

\subsubsection{Computation of raster plot and correlation coefficient of $\mathrm{Ca}^{2+}$ transient occurrence}

The times, $t_{i}$, at which transient peaks occurred are presented in a conventional raster plot.

The correlation coefficient of the calcium transients for neuron $i$ and neuron $j\left(\sigma_{C T i j}\right)$ was computed as follows: the total recording time, $T_{\text {tot }}$, was divided into $N$ intervals $(1, \ldots, \mathrm{n}, \ldots, \mathrm{N})$ of a duration $\Delta t$, that was fixed at $20 \mathrm{~s}$. Thus, if $f_{\text {in }}$ and $f_{j n}$ are the number of calcium transients of neuron $i$ and neuron $j$ in the time interval $\Delta t_{n}$, then

$\sigma_{C T_{i j}}=\frac{\sum_{n} f_{i n} f_{j n}}{\sqrt{\left(\sum_{n} f_{i n}^{2}\right)\left(\sum_{n} f_{j n}^{2}\right)}}$

so that $\sigma_{C T i j}$ depends on $\Delta t$ and varies between 0 and 1 .

\subsubsection{Statistical analysis}

Data are shown as the mean \pm standard error of the mean (s.e.m.) from the number of samples indicated in each experiment (see Results). The mean values of both morphological and activity parameters were compared among the three different conditions with One-way ANOVA test followed by Tukey post-hoc comparison, using the software SigmaPlot 10.0. Significant differences were set with $\mathrm{p}$ values inferior to $0.05(* \mathrm{p}<0.05, * * \mathrm{p}<0.01$, *** $\mathrm{p}<0.001)$.

\section{Acknowledgements}

The research leading to these results has received funding from the European Union's Seventh Framework Programme, the NEUROSCAFFOLDS Project n. 604263. The authors would like to recognize the financial support from the 3315 Innovative Teams Program of Ningbo - China. We thank Beatrice Pastore for technical assistance, Francesco Paolo Ulloa Severino for his help with calcium imaging and Manuela Schipizza Lough for carefully reading the manuscript.

\section{References}

1. Dotti, C. G., Sullivan, C. A. \& Banker, G. A. The establishment of polarity by hippocampal neurons in culture. J. Neurosci. Off. J. Soc. Neurosci. 8, 1454-1468 (1988).

2. da Silva, J. S. \& Dotti, C. G. Breaking the neuronal sphere: regulation of the actin cytoskeleton in neuritogenesis. Nat. Rev. Neurosci. 3, 694-704 (2002). 
3. Zheng, X. et al. Proteomic Analysis for the Assessment of Different Lots of Fetal Bovine Serum as a Raw Material for Cell Culture. Part IV. Application of Proteomics to the Manufacture of Biological Drugs. Biotechnol. Prog. 22, 1294-1300 (2006).

4. Arigony, A. L. V. et al. The influence of micronutrients in cell culture: a reflection on viability and genomic stability. BioMed Res. Int. 2013, 597282 (2013).

5. Kivell, B. M., McDonald, F. J. \& Miller, J. H. Serum-free culture of rat post-natal and fetal brainstem neurons. Brain Res. Dev. Brain Res. 120, 199-210 (2000).

6. Brewer, G. J., Torricelli, J. R., Evege, E. K. \& Price, P. J. Optimized survival of hippocampal neurons in B27-supplemented Neurobasal, a new serum-free medium combination. J. Neurosci. Res. 35, 567-576 (1993).

7. Beaudoin, G. M. J. et al. Culturing pyramidal neurons from the early postnatal mouse hippocampus and cortex. Nat. Protoc. 7, 1741-1754 (2012).

8. Chen, Y. et al. NS21: re-defined and modified supplement B27 for neuronal cultures. $J$. Neurosci. Methods 171, 239-247 (2008).

9. Roth, S., Zhang, S., Chiu, J., Wirth, E. K. \& Schweizer, U. Development of a serum-free supplement for primary neuron culture reveals the interplay of selenium and vitamin $\mathrm{E}$ in neuronal survival. J. Trace Elem. Med. Biol. Organ Soc. Miner. Trace Elem. GMS 24, 130-137 (2010).

10. Brewer, G. J., Boehler, M. D., Jones, T. T. \& Wheeler, B. C. NbActiv4 medium improvement to Neurobasal/B27 increases neuron synapse densities and network spike rates on multielectrode arrays. J. Neurosci. Methods 170, 181-187 (2008).

11. Cullen, D. K., Gilroy, M. E., Irons, H. R. \& Laplaca, M. C. Synapse-to-neuron ratio is inversely related to neuronal density in mature neuronal cultures. Brain Res. 1359, 44-55 (2010).

12. Araque, A. \& Navarrete, M. Glial cells in neuronal network function. Philos. Trans. R. Soc. Lond. B. Biol. Sci. 365, 2375-2381 (2010). 
13. Nedergaard, M., Ransom, B. \& Goldman, S. A. New roles for astrocytes: Redefining the functional architecture of the brain. Trends Neurosci. 26, 523-530 (2003).

14. Barker, A. J. \& Ullian, E. M. New roles for astrocytes in developing synaptic circuits. Commun. Integr. Biol. 1, 207-211 (2008).

15. Allen, N. J. \& Barres, B. A. Neuroscience: Glia - more than just brain glue. Nature 457, 675677 (2009).

16. Banker, G. A. Trophic interactions between astroglial cells and hippocampal neurons in culture. Science 209, 809-810 (1980).

17. Ivenshitz, M. \& Segal, M. Neuronal density determines network connectivity and spontaneous activity in cultured hippocampus. J. Neurophysiol. 104, 1052-1060 (2010).

18. Kaech, S. \& Banker, G. Culturing hippocampal neurons. Nat. Protoc. 1, 2406-2415 (2006).

19. Hassanpoor, H., Fallah, A. \& Raza, M. Effect of BDNF secretion by astrocyte on learning and memory: A modeling approach. in 73-76 (IEEE, 2013). doi:10.1109/ICABME.2013.6648850

20. Ebrahimi, M. et al. Astrocyte-expressed FABP7 regulates dendritic morphology and excitatory synaptic function of cortical neurons. Glia 64, 48-62 (2016).

21. Mauch, D. H. et al. CNS synaptogenesis promoted by glia-derived cholesterol. Science 294, 1354-1357 (2001).

22. Yamashita, N., Nishiyama, N., Abe, K., Saito, H. \& Fukuda, J. Primary culture of postnatal rat hypothalamic neurons in astrocyte-conditioned medium. Brain Res. 594, 215-220 (1992).

23. Nakayama, T., Momoki-Soga, T. \& Inoue, N. Astrocyte-derived factors instruct differentiation of embryonic stem cells into neurons. Neurosci. Res. 46, 241-249 (2003).

24. Todd, G. K. et al. Towards neuronal organoids: a method for long-term culturing of highdensity hippocampal neurons. PloS One 8, e58996 (2013).

25. Yan, J., Tan, T. \& Huang, Q. Protective effect of astrocyte-conditioned medium on neurons following hypoxia and mechanical injury. Chin. J. Traumatol. Zhonghua Chuang Shang Za Zhi Chin. Med. Assoc. 16, 3-9 (2013). 
26. Ulloa Severino, F. P. et al. The role of dimensionality in neuronal network dynamics. Sci. Rep. 6, 29640 (2016).

27. Cohen, E., Ivenshitz, M., Amor-Baroukh, V., Greenberger, V. \& Segal, M. Determinants of spontaneous activity in networks of cultured hippocampus. Brain Res. 1235, 21-30 (2008).

28. Hughes, E. G., Elmariah, S. B. \& Balice-Gordon, R. J. Astrocyte secreted proteins selectively increase hippocampal GABAergic axon length, branching, and synaptogenesis. Mol. Cell. Neurosci. 43, 136-145 (2010).

29. Berridge, M. J. Neuronal calcium signaling. Neuron 21, 13-26 (1998).

30. Scemes, E. \& Giaume, C. Astrocyte calcium waves: what they are and what they do. Glia 54, 716-725 (2006).

31. Puschmann, T. B. et al. Bioactive 3D cell culture system minimizes cellular stress and maintains the in vivo-like morphological complexity of astroglial cells. Glia $\mathbf{6 1}, 432-440$ (2013).

32. Zhu, Z.-H., Yang, R., Fu, X., Wang, Y.-Q. \& Wu, G.-C. Astrocyte-conditioned medium protecting hippocampal neurons in primary cultures against corticosterone-induced damages via PI3-K/Akt signal pathway. Brain Res. 1114, 1-10 (2006).

33. Hossain, S., Hewapathirane, D. S. \& Haas, K. Dynamic morphometrics reveals contributions of dendritic growth cones and filopodia to dendritogenesis in the intact and awake embryonic brain. Dev. Neurobiol. 72, 615-627 (2012).

34. Wolfes, A. C. et al. A novel method for culturing stellate astrocytes reveals spatially distinct Ca ${ }^{2+}$ signaling and vesicle recycling in astrocytic processes. J. Gen. Physiol. 149, 149-170 (2017).

35. Landis, D. M., Weinstein, L. A. \& Skordeles, C. J. Serum influences the differentiation of membrane structure in cultured astrocytes. Glia 3, 212-221 (1990).

36. Foo, L. C. et al. Development of a method for the purification and culture of rodent astrocytes. Neuron 71, 799-811 (2011). 
37. Cerbai, F. et al. The neuron-astrocyte-microglia triad in normal brain ageing and in a model of neuroinflammation in the rat hippocampus. PloS One 7, e45250 (2012).

38. Oliver, A. E., Baker, G. A., Fugate, R. D., Tablin, F. \& Crowe, J. H. Effects of Temperature on Calcium-Sensitive Fluorescent Probes. Biophys. J. 78, 2116-2126 (2000).

39. Grienberger, C. \& Konnerth, A. Imaging Calcium in Neurons. Neuron 73, 862-885 (2012).

40. Paredes, R. M., Etzler, J. C., Watts, L. T., Zheng, W. \& Lechleiter, J. D. Chemical calcium indicators. Methods 46, 143-151 (2008).

41. Bazargani, N. \& Attwell, D. Astrocyte calcium signaling: the third wave. Nat. Neurosci. 19, 182-189 (2016).

42. Amiri, M., Bahrami, F. \& Janahmadi, M. Functional contributions of astrocytes in synchronization of a neuronal network model. J. Theor. Biol. 292, 60-70 (2012).

43. Sasaki, T. et al. Astrocyte calcium signalling orchestrates neuronal synchronization in organotypic hippocampal slices. J. Physiol. 592, 2771-2783 (2014).

44. Moshtagh-Khorasani, M., Miller, E. W. \& Torre, V. The spontaneous electrical activity of neurons in leech ganglia. Physiol. Rep. 1, n/a-n/a (2013). 

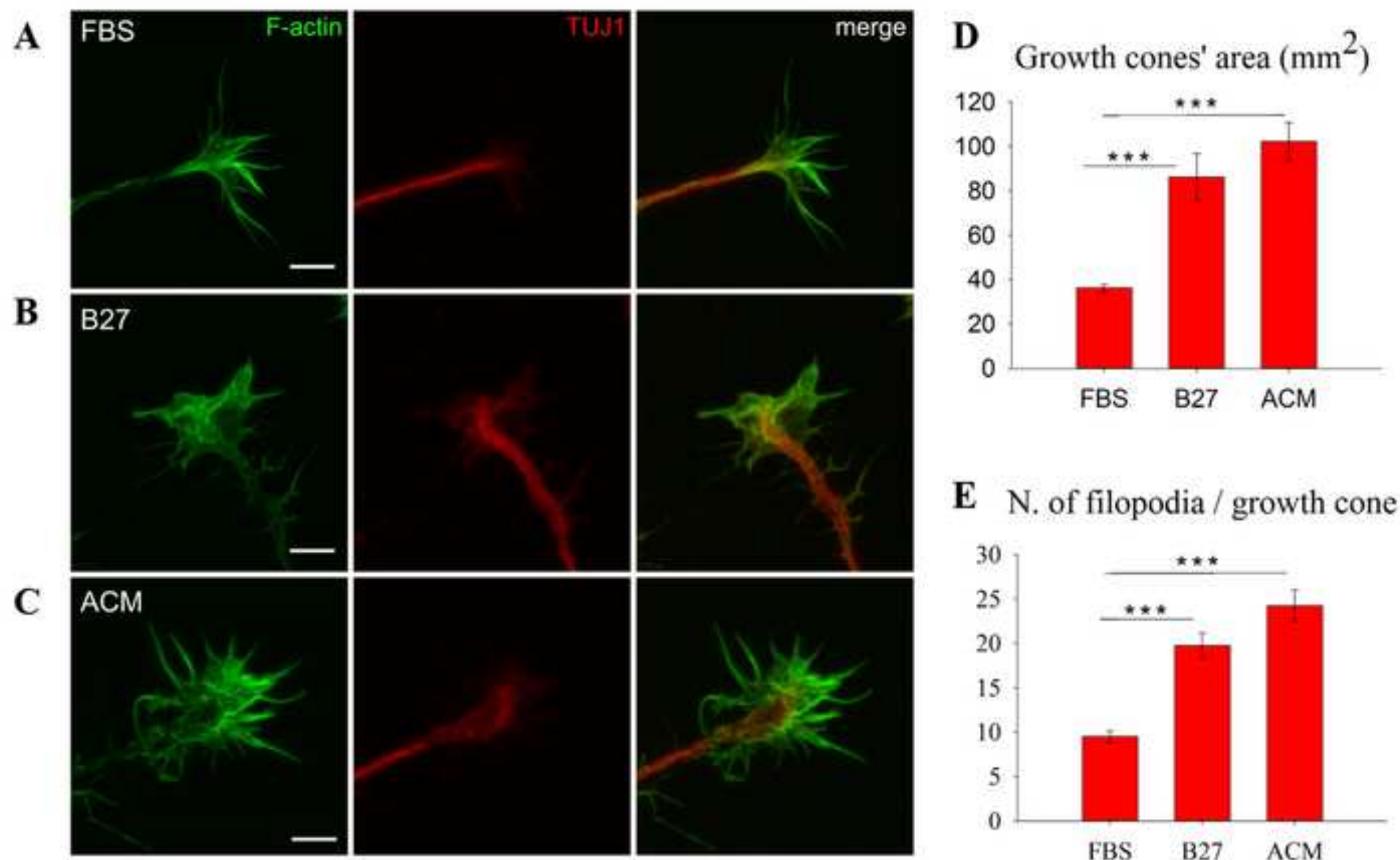

E N. of filopodia/ growth cone

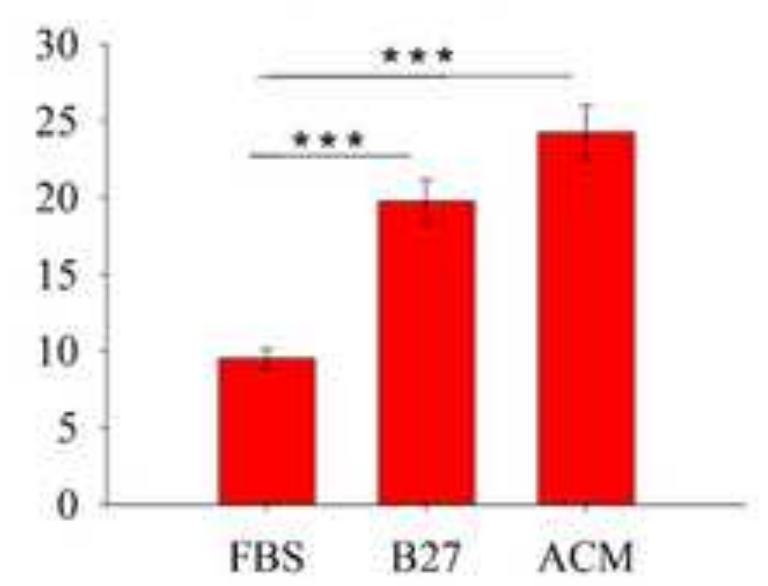



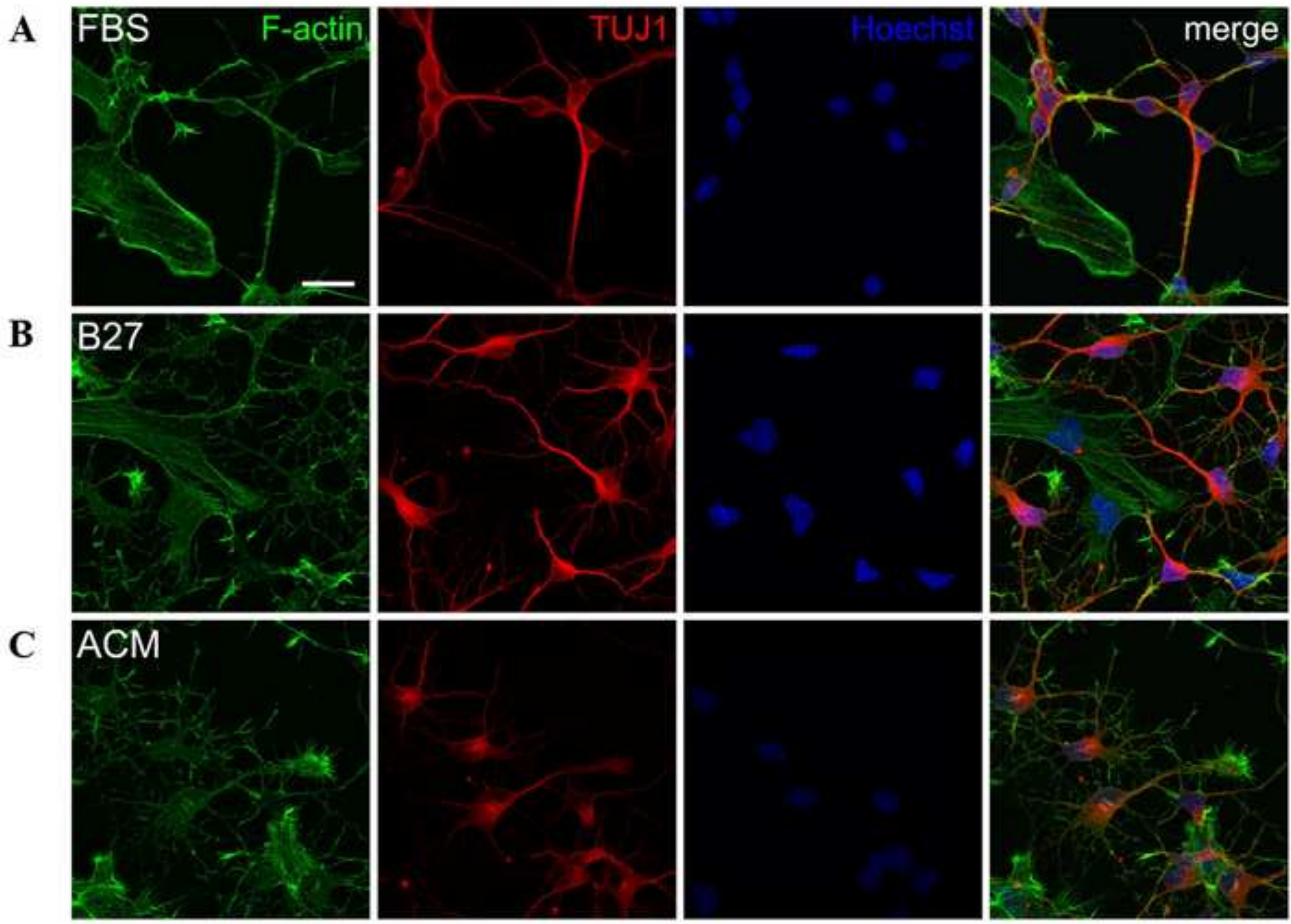
Figure 4

Click here to download high resolution image

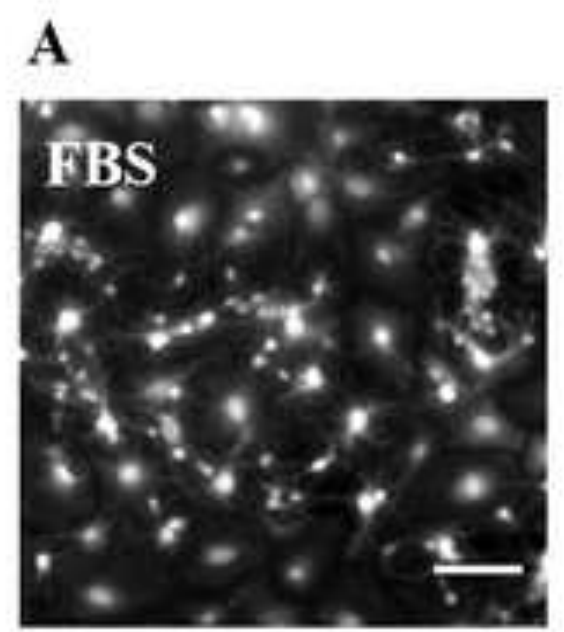

B
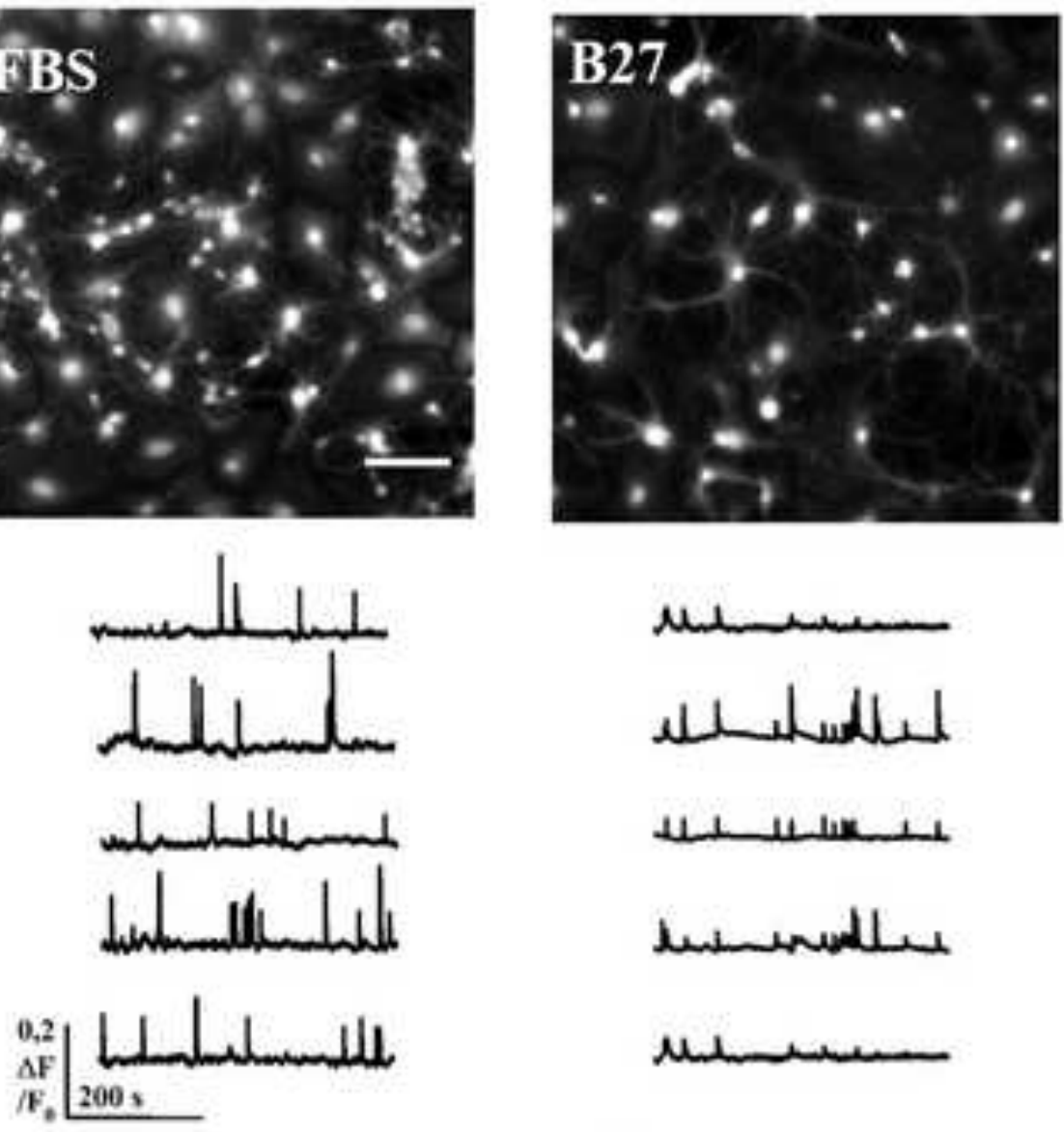

D

Peak frequency (s)

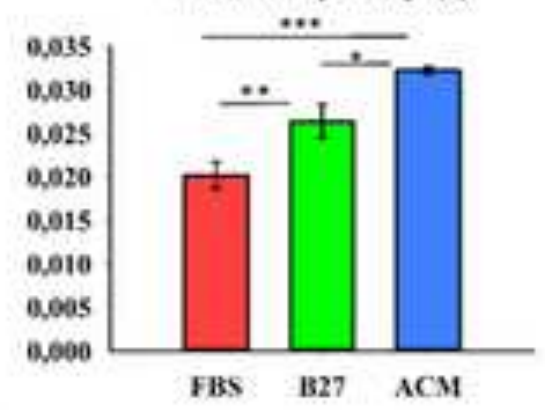

G

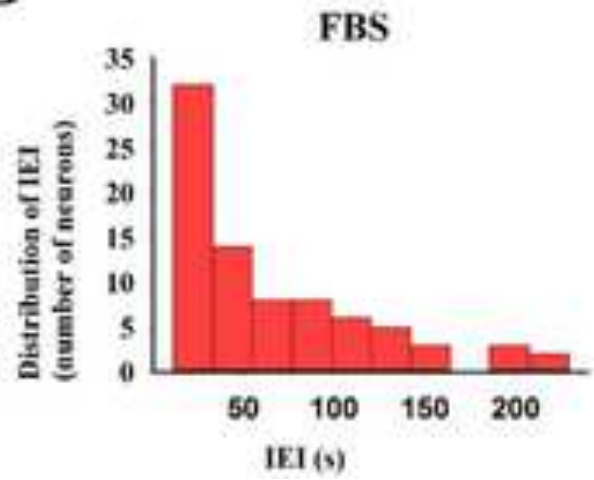

C
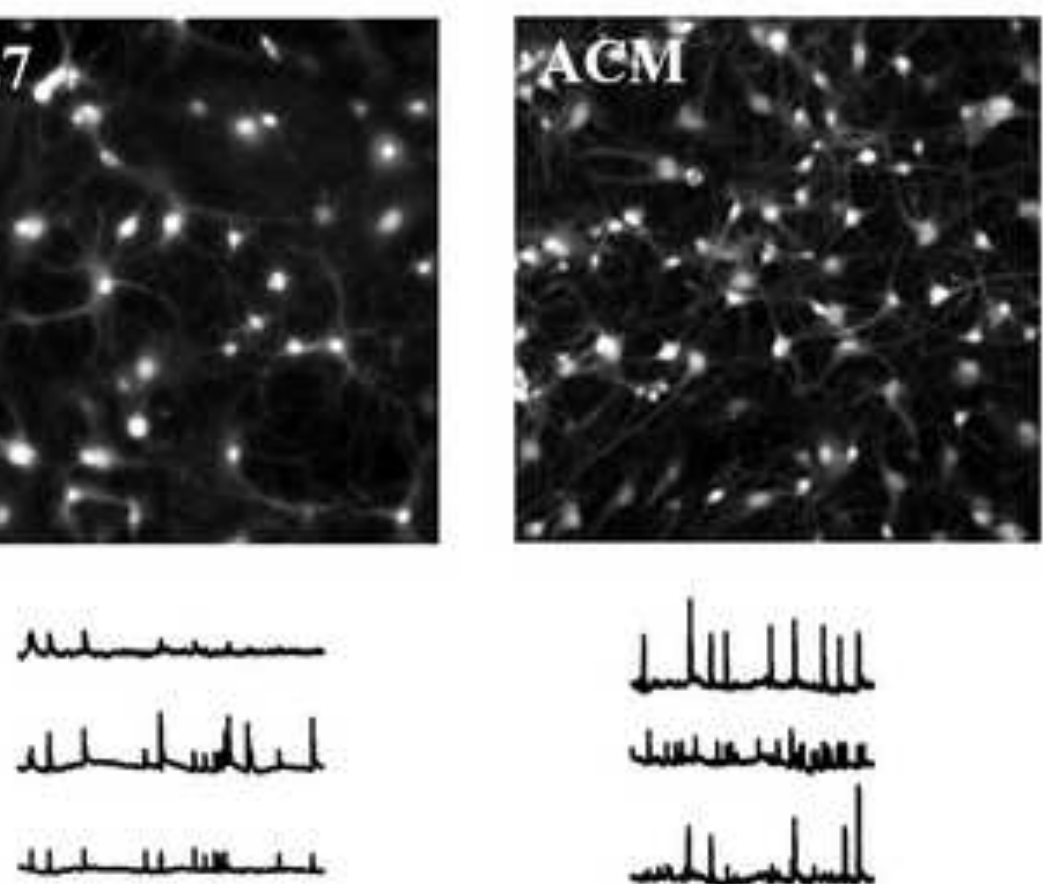

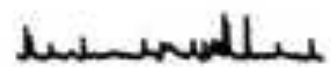

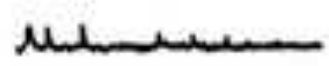

E Inter Event Interval (s)

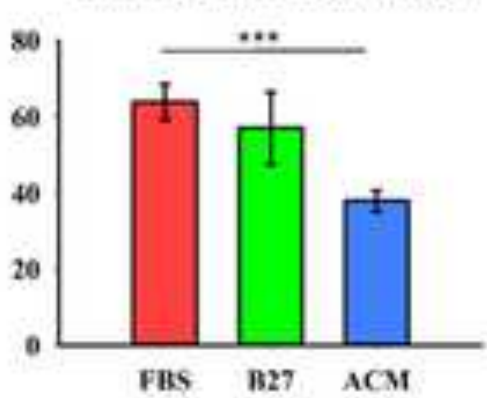

B27

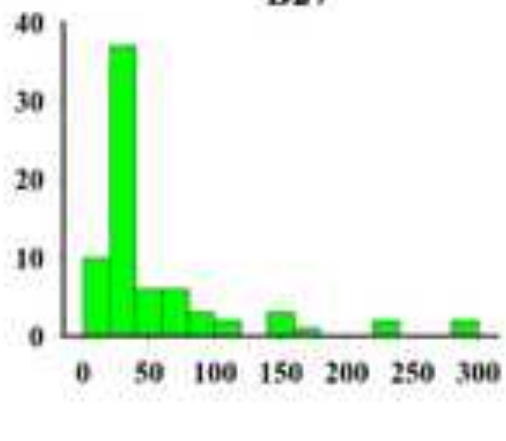

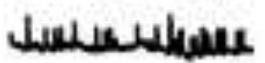

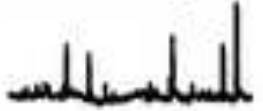

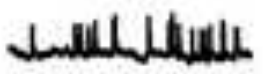

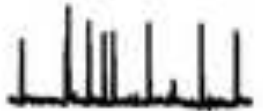

F

Cross correlation

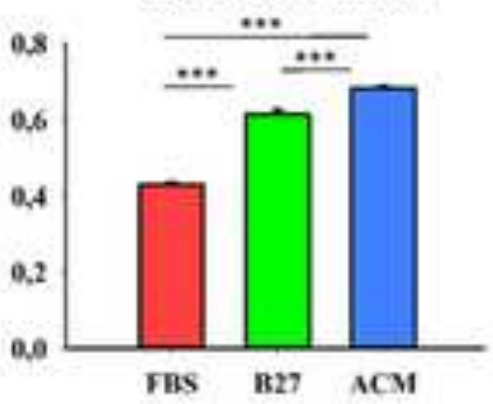

$\mathrm{ACM}$

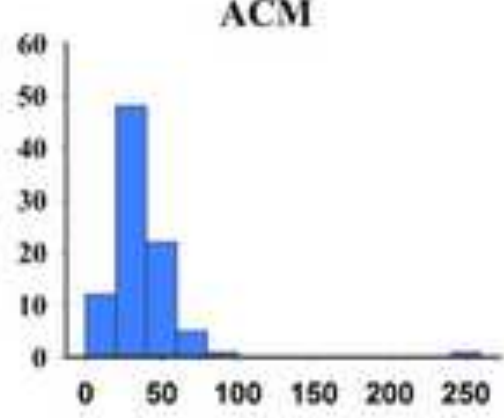


Figure 5

Click here to download high resolution image
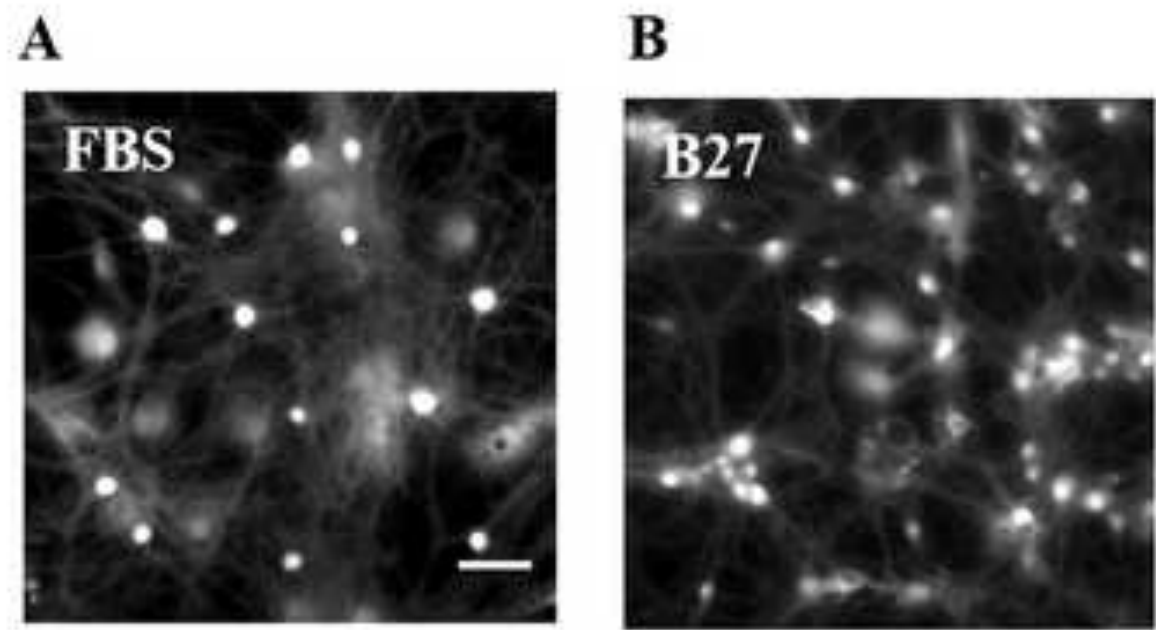

C
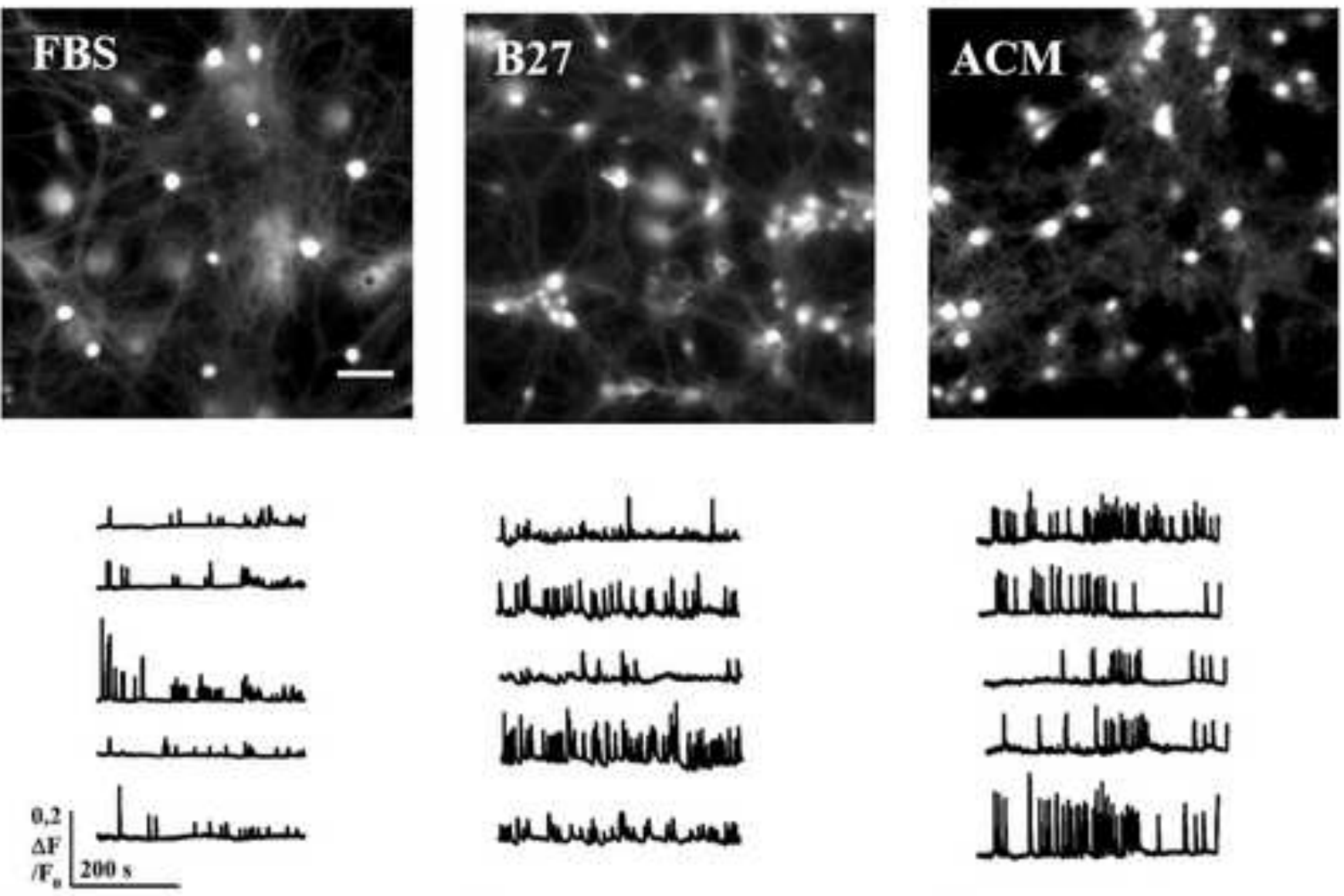

D

Peak frequency (s)

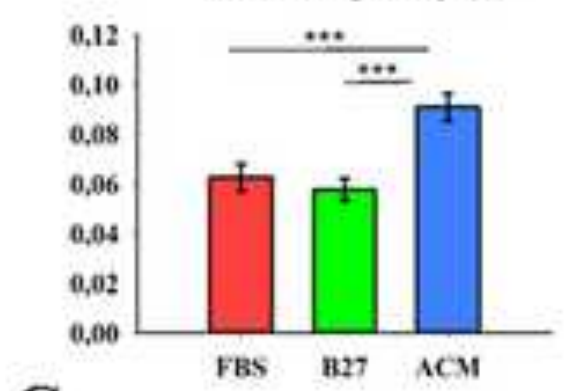

G

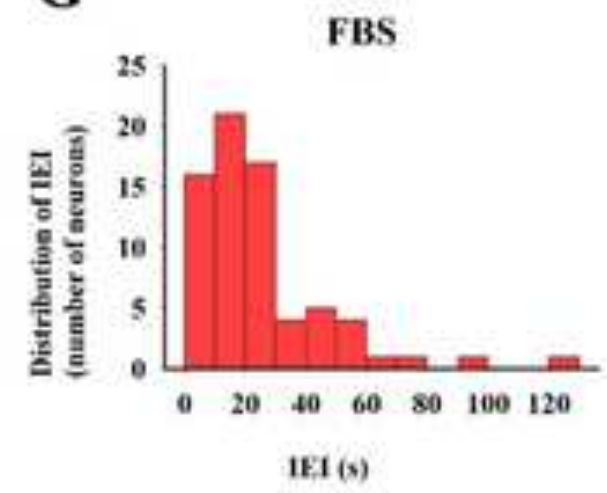

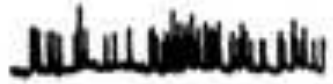
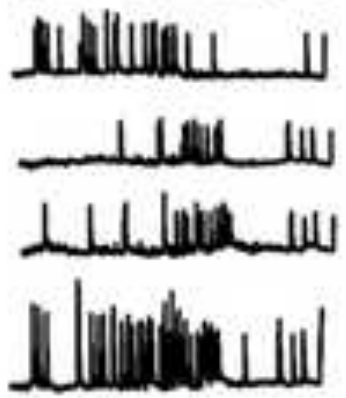

F
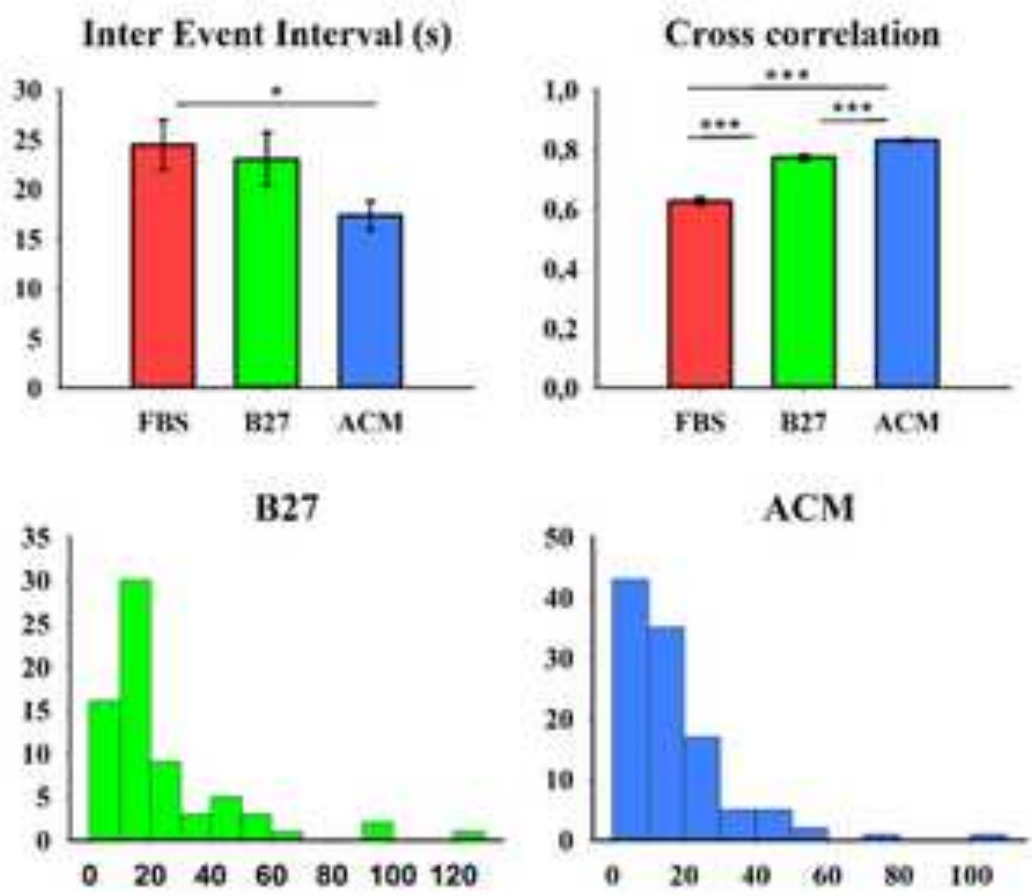

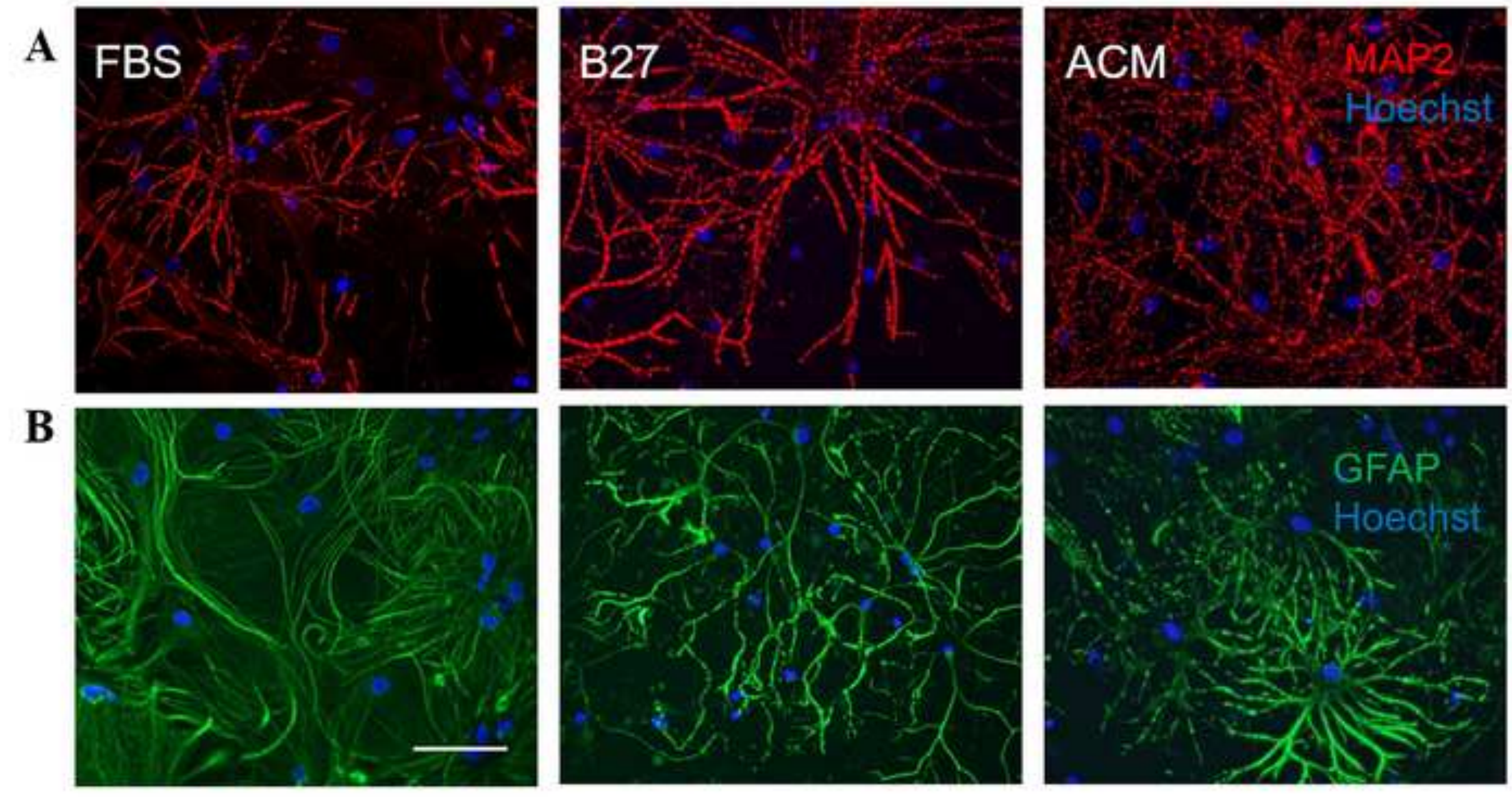

\section{Click here to download high resolution image}




\section{Click here to download Supplementary file for online publication only: Supplementary video 1.avi}

Supplementary video
Click here to downloa . 

Supplementary information Torre
Click here to download Supplemer

Supplementary information Torre
Click here to download Supplementary file for online publication only: SUPPLEMENTARY INFORMATION_Torre.docx

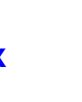

